\title{
1 TFAP2 paralogs pioneer chromatin access for MITF and directly 2 inhibit genes associated with cell migration
}

4 Colin Kenny ${ }^{1}$, Ramile Dilshat ${ }^{2}$, Hannah Seberg ${ }^{1+}$, Eric Van Otterloo ${ }^{1 *}$, Gregory

5 Bonde ${ }^{1}$, Annika Helverson ${ }^{1}$, Eiríkur Steingrímsson ${ }^{2}$, Robert A. Cornell ${ }^{1}$, \#

1. Department of Anatomy and Cell Biology, College of Medicine, University of lowa, lowa City, lowa

2. Department of Biochemistry and Molecular Biology, BioMedical Center, Faculty of Medicine, University of Iceland, Sturlugata 8, 101 Reykjavik, Iceland

+ Current address: University of Manchester

* Current address: lowa Institute for Oral Health Research, Department of Anatomy

14 and Cell Biology, Department of Periodontics, College of Dentistry, University of

15 lowa, lowa City, lowa

16 \#Corresponding author:

17 Robert-cornell@uiowa.edu, 319-335-8908

18 Conflict of interest statement: CK, RD, HS, EVO, GB, AH, ES and RAC declare no

19 conflicts of interest.

20 Key words: MITF, TFAP2A, TFAP2C, chromatin, nucleosome, pioneer factor,

21 transcriptional activation, repression. 


\section{Abstract}

Transcription factors in the Activating-enhancer-binding Protein 2 (TFAP2) family redundantly regulate gene expression in melanocytes and melanoma. Previous ChIP-seq experiments indicate that TFAP2A and Microphthalmia-associated Transcription Factor (MITF), a master regulator in these cell types, co-activate enhancers of genes promoting pigmentation. Evidence that TFAP2 paralogs can serve as pioneer factors supports the possibility that TFAP2 facilitates MITF binding at co-bound enhancers, although this model has not been tested. In addition, while MITF and TFAP2 paralogs both appear to repress genes that promote invasion, whether they do so by co-repressing enhancers is unknown. To address these questions we evaluated gene expression, chromatin accessibility, TFAP2A and MITF binding, and chromatin marks characteristic of active enhancers in SK-MEL-28 melanoma cells that were wild-type or deleted of the two TFAP2 paralogs with highest expression, TFAP2A and TFAP2C (i.e., TFAP2-KO cells). Integrated analyses revealed distinct subsets of enhancers bound by TFAP2A in WT cells that are inactivated and activated, respectively, in TFAP2-KO cells. At enhancers bound by both MITF and TFAP2A, MITF is generally lost in TFAP2A/TFAP2C double mutants, but not vice versa, implying TFAP2 pioneers chromatin access for MITF. There is a strong correlation between the sets of genes inhibited by MITF and TFAP2, although we did not find evidence that TFAP2 and MITF inhibit enhancers cooperatively. The findings imply that MITF and TFAP2 paralogs cooperatively affect the melanoma phenotype.

\section{Introduction}

Gene expression in developing melanocytes and melanoma, a cancer derived from the melanocyte lineage, is regulated by transcription factors including Microphthalmia-associated transcription factor (MITF) and members of the SOXE, PAX and TFAP2 families (Atchison, 2014; Betancur et al., 2010; Eckert et al., 2005; Goding, 2000; Hartman and Czyz, 2015; Hoek et al., 2008b; Mollaaghababa and development, and its activity is regulated at both the transcriptional and post- 
translational levels (Rambow et al., 2019). In melanoma cells, high levels of MITF activity promote cell proliferation and pigmentation, while lower levels promote an invasive phenotype (Carreira et al., 2006; Rambow et al., 2019). Mass spectroscopy revealed that MITF interacts with components of both the PBAF chromatin remodeling complex, including BRG1 and $\mathrm{CDH7}$, and the NURF remodeling complex, including RBBP4 (de la Serna et al., 2006; Laurette et al., 2015). Furthermore, chromatin immunoprecipitation of BRG1 in cells depleted of MITF revealed that MITF recruits BRG1 to the promoters of specific genes, including TYR, which encodes the rate-limiting enzyme in melanin synthesis Tyrosinase (Laurette et al., 2015). Similar analysis suggested that SOX10 also recruits BRG1 to chromatin, and at some loci it does so in co-operation with MITF (Laurette et al., 2015). Conversely, there is evidence that PAX3 inhibits the activity of MITF at the DCT promoter (Lang et al., 2005). Furthermore, low MITF activity is associated with an invasive phenotype, and deletion or knockdown of MITF results in upregulation of genes that promote migration and invasion (Dilshat et al., 2021). MITF CUT\&RUN peaks are found near some genes whose expression is upregulated in MITF mutant cells, implying MITF directly represses their expression (Dilshat et al., 2021).This set of MITF peaks is enriched for the binding site of FOXC1, a transcriptional repressor (Du et al., 2012), suggesting MITF has co-factors in its repressive function as well as its activating one.

The activating enhancer-binding family of transcription factors, comprising five members, TFAP2A-E, regulate development of many cell types and organs including neural crest, placodes, epidermis, trophectoderm, heart, kidney, and brain (Bamforth et al., 2001; Brewer et al., 2002; Knight et al., 2003; Kuckenberg et al., 2012; Luo et al., 2002; Mitchell et al., 1991; Moser et al., 1997; Schorle et al., 1996; Tan et al., 2008; Wang et al., 2006). In several contexts, including melanocyte differentiation, TFAP2 paralogs function redundantly (Kołat et al., 2021; Li and Cornell, 2007; Van Otterloo et al., 2010; Wang et al., 2008). For instance, in zebrafish tfap2a loss-offunction mutant embryos the number of melanocytes is lower than normal and pigmentation is profoundly delayed relative to in wild type embryos; this phenotype is exacerbated if tfap2a mutant embryos are also depleted of tfap2e expression with antisense morpholinos (Van Otterloo et al., 2010). In zebrafish melanoma Tfap2a and Tfap2e also appear to act redundantly to promote proliferation and, interestingly, 
to suppress cell adhesion and cell migration (Campbell et al., 2021). Consistent with redundant function of Tfap2 paralogs in the melanocyte lineage, in the skin of mouse embryos with neural-crest specific knockout of the two paralogs with highest expression, Tfap2a and Tfap2b, there are fewer-than-normal cells expressing markers of melanocytes (Seberg et al., 2017b).

Tfap2 paralogs and MITF appear to co-activate certain genes. For instance, in a human melanoma cell line, the in vitro enhancer activity of an element within an IRF4 intron depended on the simultaneous binding of MITF and TFAP2 (Praetorius et al., 2013). Further, in zebrafish tfap2a and mitfa double mutant embryos there is a greater-than-additive reduction in the number, and level of pigmentation, of melanocytes in comparison to in single mutants (Seberg et al., 2017b). Evidence that Tfap2 paralogs and Mitfa operated in parallel, rather than Tfap2 paralogs functioning upstream of mitfa expression, is that in tfap2a/tfap2e doubly-depleted zebrafish embryos, mitfa expression, and the number of mitfa-expressing cells, are not significantly changed from tfap2a singly depleted embryos (Seberg et al., 2017b). Supporting parallel activity of Tfap2 paralogs and MITF, the promoters of MITF target genes are enriched for TFAP2 consensus binding sites (Laurette et al., 2015; Rambow et al., 2015). Moreover, ChIP-seq experiments in primary melanocytes suggest that TFAP2A and MITF bind overlapping regions of chromatin near genes encoding regulators of pigmentation (Seberg et al., 2017b). Collectively, these observations indicate that TFAP2 paralogs co-activate a subset of MITF target genes by binding at the same enhancers. Still unclear, however, is whether they also corepress enhancers, and whether TFAP2 paralogs and MITF act cooperatively or independently at enhancers they co-regulate.

TFAP2 paralogs may serve as pioneer factors for MITF, although not all evidence supports this possibility. Pioneer or initiating TFs can bind nucleosome-bound DNA and recruit other TFs that lack this property called settler TFs (reviewed in Voss and Hager, 2014; Zaret, 2020; Zaret and Carroll, 2011). Evidence that TFAP2 paralogs are pioneer factors includes, first, that TFAP2 binding site is over-represented within DNase1-protected "footprints" in mouse embryonic stem cells induced to differentiate (Sherwood et al., 2014). Second, TFAP2A catalyzes assisted loading of androgen receptor (AR) in epididymis cells (Pihlajamaa et al., 2014) and estrogen receptor in 
121 MCF-7 cells (Tan et al., 2011). Third, the TFAP2 binding site is enriched for at the 122 center of ATAC-seq peaks, implying it has a strong effect on chromatin accessibility

123 (Grossman et al., 2018). Fourth, ATAC-seq peaks in naïve-stated human ESC

124 showed reduced openness in TFAP2C KO cells (Pastor et al., 2018), and forcing

125 expression of TFAP2C in human ESC is sufficient to open chromatin at loci where it

126 binds (Li et al., 2019). Finally, TFAP2A, TFAP2B and TFAP2C can bind

127 nucleosomes (Fernandez Garcia et al., 2019). Together these findings support the

128 possibility that TFAP2 displaces nucleosomes and thereby facilitates chromatin

129 binding by MITF. However, it not clear that MITF needs a pioneer factor to bind

130 chromatin. In the dynamic-assisted-loading model, all classes of TFs have short

131 residency on chromatin (reviewed in Voss and Hager, 2014). Initiating TFs are able

132 to recruit ATP-dependent chromatin remodelers (nBAF, SWI/SNF, INO80, ISWI,

133 NURD) and thereby make chromatin accessible to other TFs, i.e., the assisted TFs

134 (Swinstead et al., 2016b). As mentioned above, MITF binds various components of

135 the SWI/SNF complex (Aras et al., 2019; de la Serna et al., 2006; Keenen et al.,

136 2010) and the chromatin remodeler CHD7 (Laurette et al., 2015) and so meets the

137 criteria for an initiating factor. If the dynamic-assisted-loading model holds in this

138 situation, MITF would have no need for a pioneer factor like TFAP2 to assist its

139 binding to chromatin.

140 To address these questions, we systematically tested the effect of loss of TFAP2

141 paralogs on: nucleosome positioning, using the assay for transposase-accessible

142 chromatin using sequencing (ATAC-seq) methodology; enhancer activity, using

143 cleavage under targets and release using nuclease (CUT\&RUN) with anti-H3K27Ac,

144 anti-H3K4me3, and anti-H3K27me3; and binding of MITF, using CUT\&RUN. We

145 similarly assessed binding of TFAP2A in cells harboring loss of function mutations in

146 MITF. Our results support the notion that TFAP2 factors behave like the canonical

147 pioneer factor FOXA1: at many chromatin elements bound by TFAP2A, loss TFAP2

148 led to loss of enhancer activity, and in a large subset, it also let to chromatin

149 becoming condensed. In both of these subsets of TFAP2-activated enhancers, MITF

150 binding was TFAP2 dependent. In addition, we find evidence that TFAP2 paralogs

151 can also inhibit enhancers, and at a subset of those that they inhibit, they exclude

152 binding of MITF. Finally, the analyses suggest TFAP2 directly inhibits many of the

153 same genes that MITF inhibits, but we do not find evidence that TFAP2 and MITF 
co-repress the same enhancers. Together these findings illuminate the mechanisms by which TFAP2 and MITF coordinately regulate differentiation of melanocytes and the phenotype of melanoma cells.

Results

Tfap2a and Tfap2e redundantly promote the differentiation of zebrafish embryonic melanocytes

We first sought to use a zebrafish mutant to confirm an earlier conclusion based on morpholino-mediated knockdown that Tfap2 paralogs redundantly promote differentiation of embryonic melanophores. In the melanocyte lineage of zebrafish embryos, levels of tfap2e expression are high, those of tfap2a and tfap2c are lower ( $\sim 30 \%$ of the level of tfap2e), and those of tfap2b are negligible (Higdon et al., 2013). We previously reported that wild-type (WT) embryos injected with antisense morpholino oligonucleotides (MO) targeting the splicing of tfap2e exhibit no overt phenotype, but embryos homozygous for a tfap2a loss-of-function allele (i.e., lockjaw , Knight et al., 2003), injected with the tfap2e MO have fewer embryonic melanocytes than counterparts injected with a non-targeting control $\mathrm{MO}$, and pigmentation is delayed in them, although it occurs eventually (Van Otterloo et al., 2010). To confirm that the recovery of pigmentation did not simply reflect the transient effects of the tfap2e morpholino we used zinc-finger nucleases to engineer zebrafish lines harboring frame-shift-inducing mutations in tfap2e (details in Supplemental Fig. S1A-B). qPCR analysis showed that expression of the tfap2e transcript was significantly lower in tfap2e mutant than in WT embryos, suggesting nonsensemediated decay (Supplemental Fig. S1C). As in embryos injected with tfap2e MO, there was no overt phenotype in homozygous tfap2e mutants (Supplemental Fig. S1D-F). However, in tfap2a/tfap2e double mutants the number of melanocytes was significantly reduced in the dorsal stripe at 29 hours post fertilization (hpf) relative to those in tfap2a single mutants. At this stage these cells were under-pigmented relative to in non-mutant siblings, although their pigmentation reached wild-type levels by 48 hpf (Fig. 1A-E; Supplemental Fig. S1G-J). In summary, the reduction in melanocyte number and delay in pigmentation in tfap2a/tfap2e double mutant versus WT embryos implies that TFAP2 paralogs promote melanocyte proliferation and differentiation in a redundant fashion. 
187 We next sought to learn TFAP2 paralogs interact with MITF in activating and repressing gene expression in a single cell line. We have reported the genes differentially expressed between SK-MEL-28 melanoma cells that are WT or harboring loss-of-function mutations in all alleles of MITF, as well as binding of MITF using cleavage under targets and release under nuclease (CUT\&RUN) (Dilshat et al., 2021). Here, again using SK-MEL-28 melanoma cells, we carried out (1) CUT\&RUN using antibodies to TFAP2A (i.e., TFAP2A peaks), (2) CUT\&RUN using antibodies to chromatin marks indicative of active regulatory elements (H3K27Ac and H3K4Me3) (Creyghton et al., 2010; Pekowska et al., 2011), and of inactive chromatin (H3K27Me3) (Ringrose and Paro, 2004), and (3) ATAC-seq to distinguish between open and closed chromatin (Buenrostro et al., 2013). We used lgG as a background control and the MACS2 software to call peaks in each dataset (Supplemental Fig. S2A-B). Based on proximity to transcriptional start sites (TSS), about one-third of TFAP2A peaks appeared to be at or near promoters (within $3 \mathrm{~kb}$ of a TSS). As expected, these elements had strong H3K4Me3 signal (Supplemental Fig. S2B). At promoter-proximal TFAP2A peaks, the H3K27Ac signal in WT cells was relatively consistent, whereas at promoter-distal TFAP2A peaks the H3K27Ac signal ranged from high to background level (Supplemental Fig. S2B-C). About twothirds of TFAP2A peaks overlapped ATAC-seq peaks, indicating that they were in open chromatin (Supplemental Fig. S2D-E). Of note, the read depth (height) of a peak approximates the number of chromosome molecules where TFAP2A binds. The average read depth of the TFAP2A peaks in closed chromatin was only about $50 \%$ of that in open chromatin but was nonetheless 80 -fold higher than the $\lg$ background read depth (Supplemental Fig. S2D-E, Supplemental Fig. S3A-B for

211 example loci). Importantly, the TFAP2 binding site was strongly enriched for in both

212 TFAP2A-bound elements where the local ATAC-seq signal was called as a peak and 213 in counterparts where it was not ( $p<1 \times 10^{-1785}$ and $p<1 \times 10^{-4375}$, respectively),

214 supporting the idea that TFAP2A binds DNA directly even when the DNA is occupied

215 by nucleosomes (Supplemental Fig. S3C-D). These results indicate that TFAP2A

216 binds at both open and closed chromatin, consistent with it being a pioneer factor, 217 and at enhancers and promoters with a range of activity levels. 
219 TFAP2A activates enhancers as in pioneer factor and non-pioneer factor

\section{0 modes}

221 We next sought to identify enhancers and promoters that TFAP2 paralogs regulate directly, and, of these, the fraction that they regulate as pioneer factors. To these ends we used Crispr/Cas9 methods to introduce frame-shift mutations into the TFAP2 genes with high expression in SK-MEL-28 cells, TFAP2A and TFAP2C; we then carried out RNA-seq, ATAC-seq, and CUT\&RUN with antibodies to H3K27Ac, H3K4me3, and H3K27me3. In two independent knockout clones (hereafter, TFAP2KO cells), Western blot analysis showed an absence of immunoreactivity for both proteins (Supplemental Fig. S4A-E). Control clones (hereafter WT cells) were derived from the parental SK-MEL-28 line transiently transfected with Cas9 but not with guide RNAs. RNA-seq revealed that expression of 532 genes was downregulated, and expression of 609 genes was upregulated, in TFAP2-KO cells

232 (i.e., in both clones) versus in WT cells (Supplemental Fig. S5A volcano plot). We will refer to these sets as "TFAP2-activated genes" and "TFAP2-inhibited genes," respectively.

To identify candidates for enhancers directly activated by TFAP2 paralogs we first filtered TFAP2A peaks for those in chromatin that was open and active in WT cells (i.e., coinciding with peaks of ATAC-seq and H3K27Ac) $(21,745 / 36,948$ of TFAP2A peaks), then for those greater than $1 \mathrm{~kb}$ from a transcription start site (i.e., to filter out promoters) $(11,005 / 21,745)$, and finally for those where the local H3K27Ac signal was significantly lower (adj $p<0.05, \log 2 F C<-1$ ) in TFAP2-KO cells relative to in WT cells $(3,858 / 11,005)$.

To determine how often TFAP2 activates enhancers as a pioneer factor, at each directly TFAP2-activated enhancer we evaluated the ATAC-seq signal in WT and TFAP2-KO cells. At about half of the enhancers the ATAC-seq signal was also significantly lower (adj $\mathrm{p}<0.05$, log2FC <-1) in TFAP2-KO versus in WT cells (i.e., the ATAC-seq signal was TFAP2-activated) (Fig. 2A, E-E'); at this subset we infer that TFAP2 paralogs function as pioneer factors. At the remaining half, the ATAC- 
signal was TFAP2-independent) (Fig. 2B, F-F'); at this subset we infer that TFAP2

250 paralogs do not function as pioneer factors but rather as a transcriptional activator.

251 Consistent with both subsets indeed being enhancers activated by TFAP2, both

252 were associated with TFAP2-activated genes. Interestingly, the association was

253 stronger for those where TFAP2 functions as a pioneer factor (Fig. 2 I, J) (Table 1

254 and Table 2). Moreover, at both subsets the H3K4me3 signal, which is associated

255 with enhancer activity (Pekowska et al., 2011), was reduced in TFAP2-KO cells

256 relative to in WT cells (Fig. 2E', F'"). While both subsets were strongly enriched for

257 the TFAP2 binding site and certain other binding sites (e.g., RUNX), the subset

258 pioneered by TFAP2 was more strongly enriched for the SOXE and MITF binding

259 sites, while the non-pioneered subset was more strongly enriched for the FRA1,

260 TEAD and the ZFX binding sites (Fig. 2M, N). Of note, FRA1 is a pioneer factor (Lee

261 et al., 2018) which could explain why these elements do not depend on TFAP2 to be

262 free of nucleosomes.

Table 1: Hypergeometric analysis: TFAP2 regulated enhancers and gene expression

\begin{tabular}{|c|c|c|c|c|c|c|c|c|c|}
\hline $\begin{array}{l}\text { Regulatory } \\
\text { element } \\
\text { (Enhancer) }\end{array}$ & $\begin{array}{l}\text { Effect of } \\
\text { TFAP2 on } \\
\text { H3K27Ac }\end{array}$ & $\begin{array}{l}\text { Effect of } \\
\text { TFAP2 on } \\
\text { ATAC }\end{array}$ & $\begin{array}{l}\text { Effect of } \\
\text { TFAP2 on } \\
\text { RNA-seq }\end{array}$ & $\begin{array}{l}\mathrm{N} \text { (\# of } \\
\text { elements) }\end{array}$ & OR & LB & UB & $\begin{array}{l}\text { p-value } \\
\text { All DEG's }\end{array}$ & $\begin{array}{l}\text { p-value } \\
\mid \text { Log2FC } \mid>1\end{array}$ \\
\hline- & Activates & - & Activates & 3,838 & 2.36 & 2.06 & 2.70 & $2.8 \times 10^{-33}$ & $1.12 \times 10^{-14}$ \\
\hline- & Activates & - & Inhibits & 3,838 & 1.03 & 0.85 & 1.24 & $7.0 \times 10^{-01}$ & $5.9 \times 10^{-01}$ \\
\hline- & Inhibits & - & Activates & 1,304 & 1.23 & 0.93 & 1.60 & $1.24 \times 10^{-1}$ & $7.1 \times 10^{-01}$ \\
\hline- & Inhibits & - & Inhibits & 1,304 & 2.30 & 1.87 & 2.82 & $4.95 \times 10^{-14}$ & $4.8 \times 10^{-06}$ \\
\hline Pioneered & Activates & Activates & Activates & 2,002 & 2.60 & 2.23 & 3.06 & $5.44 \times 10^{-29}$ & $6.3 \times 10^{-20}$ \\
\hline Pioneered & Activates & Activates & Inhibits & 2,002 & 1.08 & 0.80 & 1.25 & $9.0 \times 10^{-01}$ & $8.5 \times 10^{-01}$ \\
\hline $\begin{array}{l}\text { Non- } \\
\text { Pioneered }\end{array}$ & Activates & Independent & Activates & 1,836 & 1.90 & 1.57 & 2.30 & $2.45 \times 10^{-10}$ & $1.0 \times 10^{-06}$ \\
\hline $\begin{array}{l}\text { Non- } \\
\text { Pioneered }\end{array}$ & Activates & Independent & Inhibits & 1,836 & 1.05 & 0.82 & 1.32 & $6.33 \times 10^{-01}$ & $2.8 \times 10^{-01}$ \\
\hline Pioneered & Inhibits & Inhibits & Activates & 864 & 0.68 & 0.33 & 1.251 & $2.9 \times 10^{-01}$ & $1.0 \times 10^{-00}$ \\
\hline Pioneered & Inhibits & Inhibits & Inhibits & 864 & 2.33 & 1.61 & 3.28 & $8.08 \times 10^{-06}$ & $9.44 \times 10^{-05}$ \\
\hline $\begin{array}{l}\text { Non- } \\
\text { Pioneered }\end{array}$ & Inhibits & Independent & Activates & 440 & 1.33 & 0.83 & 2.05 & 0.17 & 0.83 \\
\hline $\begin{array}{l}\text { Non- } \\
\text { Pioneered }\end{array}$ & Inhibits & Independent & Inhibits & 440 & 1.72 & 1.34 & 2.43 & 0.008 & 0.23 \\
\hline
\end{tabular}

OR: odds ratio, LB: lower boundary, UB: upper boundary, ALL DEGs: all differentially expressed genes (FDR < 0.05$)$ in TFAP2-KO clones (two independent clones, 4 replicates each) and WT (4 replicates) SK-MEL-28 cells. Log2FC: Log base 2 
Table 2: Hypergeometric analysis: TFAP2-activated enhancer and promoters, and TFAP2-activated gene expression

\begin{tabular}{|c|c|c|c|c|c|c|c|c|}
\hline $\begin{array}{l}\text { Enhancers and } \\
\text { promoters }\end{array}$ & $\begin{array}{l}\text { Effect of } \\
\text { TFAP2 on } \\
\text { H3K27Ac }\end{array}$ & $\begin{array}{l}\text { Effect of } \\
\text { TFAP2 on } \\
\text { ATAC }\end{array}$ & $\begin{array}{l}\text { Effect of } \\
\text { TFAP2 on } \\
\text { RNA-seq }\end{array}$ & $\begin{array}{l}\mathrm{N} \text { (\# of } \\
\text { elements) }\end{array}$ & OR & LB & UB & $\begin{array}{l}\text { p-value } \\
\text { Log2FC < - } 1\end{array}$ \\
\hline All peaks & - & - & Activates & 36,948 & 1.66 & 1.40 & 1.98 & $2.4 \times 10^{-08}$ \\
\hline $\begin{array}{l}\text { TFAP2A } \\
\text { (open } \\
\text { chromatin) }\end{array}$ & - & - & Activates & 26,373 & 1.62 & 1.36 & 1.92 & $1.6 \times 10^{-08}$ \\
\hline $\begin{array}{l}\text { TFAP2A } \\
\text { peaks }\end{array}$ & Activates & - & Activates & 4,601 & 3.11 & 2.57 & 3.9 & $6.0 \times 10^{-24}$ \\
\hline $\begin{array}{l}\text { TFAP2A } \\
\text { peaks }\end{array}$ & Activates & Independent & Activates & 2,324 & 2.75 & 2.10 & 3.55 & $2.1 \times 10^{-12}$ \\
\hline $\begin{array}{l}\text { TFAP2A } \\
\text { peaks }\end{array}$ & - & Activates & Activates & 5,443 & 2.58 & 2.3 & 2.89 & $3.9 \times 10^{-27}$ \\
\hline $\begin{array}{l}\text { TFAP2A } \\
\text { peaks }\end{array}$ & Independent & Activates & Activates & 3,241 & 2.97 & 2.73 & 3.70 & $8.8 \times 10^{-19}$ \\
\hline $\begin{array}{l}\text { TFAP2A } \\
\text { peaks }\end{array}$ & Activates & Activates & Activates & 2,202 & 4.4 & 3.46 & 5.58 & $4.1 \times 10^{-27}$ \\
\hline $\begin{array}{l}\text { TFAP2A } \\
\text { peaks }\end{array}$ & Activates OR & R Activates & Activates & 7,842 & 3.80 & 3.1 & 4.60 & $1.44 \times 10^{-35}$ \\
\hline $\begin{array}{l}\text { TFAP2A } \\
\text { peaks }\end{array}$ & Independent & Independent & Activates & 19,570 & 1.55 & 1.39 & 1.73 & $6.1 \times 10^{-02}$ \\
\hline
\end{tabular}

OR: odds ratio, LB: lower boundary, UB: upper boundary, Log2FC: Log base 2 fold change. Differentially expressed genes in TFAP2-KO clones (two independent clones, 4 replicates each) and WT (4 replicates) SK-MEL-28 cells. N: numbers of TFAP2 regulated enhancer and promoter peaks used in the analysis.

266 Because of evidence that TFAP2A directly represses gene expression (Lin et al.,

267 2016; Liu et al., 2007; Wong et al., 2012) we next sought to identify candidates for 268 enhancers directly inhibited by TFAP2 paralogs. To this end we filtered promoter-

269 distal TFAP2A peaks for those where the local H3K27Ac signal was higher in

270 TFAP2-KO cells than in WT cells (adj. $p<0.05$, log2FC $>1$ ). Analogously to TFAP2-

271 activated enhancers, candidate TFAP2-inhibited enhancers were split between a

272 subset where the ATAC-seq signal was higher in TFAP2-KO cells than in WT cells

273 (i.e., TFAP2-inhibited) (Fig. 2C, G-G') and a subset where it was TFAP2-

274 independent (Fig. 3D, H-H'). The first subset was significantly associated with

275 TFAP2-inhibited genes (Fig. 3K, Table 1 and Table 3) and the average H3K4me3

276 signal at these sites was TFAP2-inhibited (Fig. 3G'). Because TFAP2 concomitantly

277 inhibits enhancer activity and chromatin accessibility, we define these enhancers as

278 inhibited by TFAP2 in pioneer factor-mode. The binding site for TFAP2 site was

279 strongly enriched for in these sites, as were those for ETS1 and CTCF (Fig. 30),

280 both transcriptional repressors (Kim et al., 2015; Mavrothalassitis and Ghysdael,

281 2000). By contrast, the subset of candidate TFAP2-inhibited enhancers where the 
ATAC-signal was TFAP2-independent was not associated with TFAP2-inhibited genes (Fig. 3L, Table 1), and the average H3K4me3 signal at them was TFAP2 independent (Fig. 3H"). We infer these elements are not TFAP2-inhibited enhancers, despite having elevated H3K27Ac signal in TFAP2-KO cells in comparison to WT cells. In conclusion, at TFAP2-inhibited enhancers TFAP2 recruits machinery that condenses chromatin and inhibits enhancer activity; the canonical pioneer factor FOXA1 also has this potential (Sekiya and Zaret, 2007; Watts et al., 2011).

Table 3: Hypergeometric analysis: TFAP2-inhibited enhancers and promoters, and TFAP2-inhibited gene expression

\begin{tabular}{|c|c|c|c|c|c|c|c|c|}
\hline $\begin{array}{l}\text { Enhancers and } \\
\text { promoters }\end{array}$ & $\begin{array}{l}\text { Effect of } \\
\text { TFAP2 on } \\
\text { H3K27Ac }\end{array}$ & $\begin{array}{l}\text { Effect of } \\
\text { TFAP2 on } \\
\text { ATAC } \\
\end{array}$ & $\begin{array}{l}\text { Effect of } \\
\text { TFAP2 on } \\
\text { RNA-seq }\end{array}$ & $\begin{array}{l}\mathrm{N} \text { (\# of } \\
\text { elements) }\end{array}$ & OR & LB & UB & $\begin{array}{l}\text { p-value } \\
\mid \text { Log } 2 F C \mid>1\end{array}$ \\
\hline $\begin{array}{l}\text { TFAP2A } \\
\text { (closed } \\
\text { chromatin) }\end{array}$ & - & - & Inhibits & 12,931 & 1.39 & 1.15 & 1.69 & $8.0 \times 10^{-04}$ \\
\hline $\begin{array}{l}\text { TFAP2A } \\
\text { (open } \\
\text { chromatin) }\end{array}$ & - & - & Inhibits & 26,373 & 1.06 & 0.90 & 1.25 & $4.8 \times 10^{-01}$ \\
\hline $\begin{array}{l}\text { TFAP2A } \\
\text { peaks }\end{array}$ & Inhibits & - & Inhibits & 2,830 & 1.68 & 1.31 & 2.13 & $5.1 \times 10^{-05}$ \\
\hline $\begin{array}{l}\text { TFAP2A } \\
\text { peaks }\end{array}$ & - & Inhibits & Inhibits & 4,236 & 1.69 & 1.34 & 2.12 & $1.33 \times 10^{-05}$ \\
\hline $\begin{array}{l}\text { TFAP2A } \\
\text { peaks }\end{array}$ & Inhibits & Inhibits & Inhibits & 1,695 & 2.06 & 1.51 & 2.76 & $8.47 \times 10^{-06}$ \\
\hline $\begin{array}{l}\text { TFAP2A } \\
\text { peaks }\end{array}$ & Inhibits & Inhibits & Inhibits & 5,371 & 1.68 & 1.38 & 2.03 & $3.61 \times 10^{-07}$ \\
\hline $\begin{array}{l}\text { TFAP2A } \\
\text { peaks }\end{array}$ & Independent & Independent & Inhibited & 21,848 & 0.98 & 0.82 & 1.15 & $7.0 \times 10^{-01}$ \\
\hline
\end{tabular}

OR: odds ratio, LB: lower boundary, UB: upper boundary, Log2FC: Log base 2 fold change. Differentially expressed genes in TFAP2-KO clones (two independent clones, 4 replicates each) and WT (4 replicates) SK-MEL-28 cells. N: numbers of TFAP2 regulated enhancer and promoter peaks used in the analysis.

291 We similarly analyzed whether and how TFAP2 directly activates or directly inhibits 292 promoters. Although TFAP2A peaks are frequently found at promoters (8277 genes 293 have a TFAP2A peak within $1 \mathrm{~kb}$ of the TSS), it was uncommon for the underlying 294 H3K27Ac and H3K4Me3 signal to be elevated or reduced in TFAP2 KO cells relative 295 to in WT cells (119 and 31 candidate for directly activated and directly inhibited 296 promoters, respectively). Similar to the trends for TFAP2-regulated enhancers, the 297 pioneered subset of TFAP2-activated promoters was more strongly associated with 298 TFAP2-activated genes than the non-pioneered subset, and only the pioneered 299 subset of TFAP2-inhibited promoters was associated with TFAP2-inhibited genes

300 (Supplemental Fig. S6A-L; additional examples in Supplemental Fig. S7A-B). 
302 At additional subsets of MITF peaks overlapping TFAP2A peaks, TFAP2

303 facilitates chromatin access for MITF both in pioneer and non-pioneer factor modes

A prediction of the TFAP2-as-pioneer-factor model is that binding of transcription factors, like MITF, will depend on TFAP2. Among 37,643 MITF peaks in WT SKMEL-28 cells that we previously identified by CUT\&RUN (Dilshat et al., 2021), 15,752 (42\%) overlap a TFAP2A peak (i.e., assessed in this study). Of these, 9,413 $(60 \%)$ were within open and active chromatin (Supplemental Fig. S8A). To assess MITF binding in the absence of TFAP2 we carried out anti-MITF CUT\&RUN in TFAP2-KO cell lines. Of note, as MITF RNA levels in TFAP2-KO cells are only about $60 \%$ of those in WT cells, an across the board decrease in the average height (read depth) of MITF peaks was possible. Instead, we observed that the average height of MITF peaks not overlapping TFAP2A peaks was equivalent in TFAP2-KO cells and in WT (Supplemental Fig. S8B). By contrast, among MITF peaks overlapping TFAP2A peaks $(15,752)$, the height of 5,443 (35\%) was significantly lower in TFAP2KO cells than in WT cells (adj. $p<0.05, \log 2 F C<-1$ ). (Fig. 3A-D; Supplemental

318 Fig. S8C-D and Supplemental Fig. S9). We refer to these as "TFAP2-dependent

319 MITF peaks," referring only to MITF peaks that appear to be directly TFAP2-

320 dependent (because they overlap TFAP2A peaks in WT cells).

322 We reasoned that TFAP2 paralogs could facilitate MITF binding by displacing

323 nucleosomes (i.e., in pioneer factor mode) or alternatively by elevating MITF's affinity

324 for open DNA. Consistent with both models, we observed that TFAP2-dependent

325 MITF peaks were in three subsets with respect to the TFAP2-dependence of the underlying ATAC-seq signal. At about $57 \%(3,083 / 5,443)$ the ATAC-seq signal was significantly lower (Fig. 3B-C, 3F-F'), at 37\% $(2,022 / 5,443)$ it was unchanged (Fig. 3D, 3G-G'), and at 6\% it was higher (Fig. 3E, 3H-H') in TFAP2-KO cells than in WT cells. The first two subsets were strongly associated with TFAP2-activated genes

330 (Hypergeometric test; $p$-value $=8.4 \times 10^{-26}$ and $p$-value $=1.07 \times 10^{-13}$ respectively) 331 and with MITF-activated genes (Hypergeometric test; $p$-value $=1.16 \times 10^{-21}$ and $p$ 332 value $=4.3 \times 10^{-11}$ respectively) (Table 4). We infer that at the first subset, TFAP2 is 
333 a pioneer factor, facilitating access for MITF and other transcription factors

334 (illustrated in Fig. 3F'). Supporting this prediction, the transcription factor binding

335 sites for MITF, SOX10, RUNX and FRA1 were strong enriched at such elements

336 (Fig. 3F"'). At the second subset, TFAP2 is a transcriptional activator that recruits

337 MITF, also functioning as a transcriptional activator; we presume another protein

338 serves as a pioneer factor at this subset (illustrated in Fig. 3G'). Consistent with this

339 notion, the binding site for JUN, a widely deployed pioneer factor (Vierbuchen et al.,

340 2017), site is strongly enriched in these elements (Fig. 3G'”). Examples are shown

341 of TFAP2-dependent MITF peaks near FRMD4B, CYP7B1, TRPM1 SOX9, EDNRB,

342 MREG, GPR143, SNAI2, MEF2C, MYO5A, PAX3, EN1 and FOXI3 genes (Fig. 3B-D

343 and Supplemental Fig. S8D). At the third subset of TFAP2-dependent MITF peaks,

344 where ATAC-seq signal was higher in TFAP2-KO cells than in WT cells

345 (Supplemental Fig. S10A-A'), TFAP2 may serve as a pioneer factor for MITF in

346 MITF's proposed role as transcriptional repressor (Dilshat et al., 2021) (illustrated in

347 Supplemental Fig. S10A"). However, this category of element was not enriched

348 near genes inhibited by either TFAP2 or MITF (Hypergeometric test; $p$-value $=6.02 \mathrm{x}$

$34910^{-02}$ and $p$-value $=9.12 \times 10^{-02}$ respectively). These results are consistent with

350 TFAP2 facilitating access for MITF, in its transcriptional activator form, to enhancers

351 in both pioneer-factor and non-pioneer factor modes. 
Table 4: Hypergeometric analysis: TFAP2-dependent MITF peaks and gene expression

\begin{tabular}{|c|c|c|c|c|c|c|c|c|}
\hline $\begin{array}{l}\text { TFAP2's } \\
\text { effect on: } \\
\text { MITF }\end{array}$ & $\begin{array}{l}\text { Effect of } \\
\text { TFAP2 on } \\
\text { ATAC }\end{array}$ & $\begin{array}{l}\text { Effect of } \\
\text { TFAP2 on } \\
\text { RNA-seq }\end{array}$ & $\begin{array}{l}\mathrm{N} \text { (\# of } \\
\text { elements) }\end{array}$ & OR & LB & UB & $\begin{array}{l}\text { p-value } \\
\text { All DEG's }\end{array}$ & $\begin{array}{l}\text { p-value } \\
\mid \text { Log2FC| }>1\end{array}$ \\
\hline $\begin{array}{l}\text { TFAP2- } \\
\text { dependent }\end{array}$ & - & Activates & 5,443 & 2.96 & 2.56 & 3.43 & $3.47 \times 10^{-41}$ & $8.4 \times 10^{-26}$ \\
\hline $\begin{array}{l}\text { TFAP2- } \\
\text { dependent }\end{array}$ & - & Inhibits & 5,443 & 1.00 & 0.81 & 1.23 & $9.5 \times 10-01$ & $7.2 \times 10^{-01}$ \\
\hline $\begin{array}{l}\text { TFAP2- } \\
\text { dependent }\end{array}$ & Activates & Activates & 3,083 & 3.43 & 2.95 & 3.98 & $3.19 \times 10^{-49}$ & $1.16 \times 10^{-26}$ \\
\hline $\begin{array}{l}\text { TFAP2- } \\
\text { dependent }\end{array}$ & Activates & Inhibits & 3,083 & 1.15 & 0.92 & 1.42 & $1.7 \times 10^{-01}$ & $4.0 \times 10^{-01}$ \\
\hline $\begin{array}{l}\text { TFAP2- } \\
\text { dependent }\end{array}$ & Independent & Activates & 2,358 & 2.38 & 1.81 & 2.55 & $1.6 \times 10^{-16}$ & $1.07 \times 10^{-13}$ \\
\hline $\begin{array}{l}\text { TFAP2- } \\
\text { dependent }\end{array}$ & Independent & Inhibits & 2,358 & 1.13 & 0.91 & 1.40 & $2.3 \times 10^{-01}$ & $4.0 \times 10^{-01}$ \\
\hline $\begin{array}{l}\text { Mutually } \\
\text { dependent }\end{array}$ & Activates & Activates & 717 & 2.967 & 1.768 & 4.756 & $3.47 \times 10^{-05}$ & $9.28 \times 10^{-05}$ \\
\hline $\begin{array}{l}\text { Mutually } \\
\text { dependent }\end{array}$ & Activates & Inhibits & 717 & 0.789 & 0.284 & 1.772 & $7.07 \times 10^{-01}$ & 1.00 \\
\hline $\begin{array}{l}\text { TFAP2- } \\
\text { inhibited }\end{array}$ & Inhibits & Activates & 924 & 0.73 & 0.48 & 1.06 & $1.11 \times 10^{-01}$ & 1.00 \\
\hline $\begin{array}{l}\text { TFAP2- } \\
\text { inhibited }\end{array}$ & Inhibits & Inhibits & 924 & 2.75 & 2.21 & 3.39 & $1.1 \times 10^{-17}$ & $3.5 \times 10^{-04}$ \\
\hline $\begin{array}{l}\text { TFAP2- } \\
\text { inhibited }\end{array}$ & Independent & Activates & 681 & 1.18 & 0.80 & 1.68 & 1.00 & 1.00 \\
\hline $\begin{array}{l}\text { TFAP2- } \\
\text { inhibited }\end{array}$ & Independent & Inhibits & 681 & 2.27 & 1.72 & 2.94 & $2.9 \times 10^{-08}$ & $1.2 \times 10^{-02}$ \\
\hline
\end{tabular}

OR: odds ratio, LB: lower boundary, UB: upper boundary, ALL DEGs: all differentially expressed genes in TFAP2-KO clones (two independent clones, 4 replicates each) and WT (4 replicates) SK-MEL-28 cells. Log2C: Log base 2 fold change. N: numbers of TFAP2-dependent MITF peaks used in the analysis.

354 To test for the converse dependence, we carried out anti-TFAP2A CUT\&RUN in

355 MITF-KO cells. TFAP2A mRNA levels were equivalent in MITF-KO and WT cells,

356 and the average TFAP2A peak height was globally equivalent by CUT\&RUN. At $13 \%$

357 (717/ 5334) of TFAP2-dependent MITF peaks, the TFAP2A peak was, reciprocally,

358 significantly reduced in MITF-KO cells (Fig. 3A, C). At such loci, the average ATAC-

359 seq was reduced in TFAP2-KO cells than in WT cells (Fig. 3C, 3I-I'). We termed

360 these peaks mutually-dependent (illustrated in Fig. 31'). Interestingly, mutually-

361 dependent MITF/ TFAP2 peaks were enriched in binding motifs for TFAP2, MITF,

362 BRM2 and TEAD4 but, notably and unlike the other subsets of TFAP2-dependent

363 MITF peaks, not for SOXE (Fig. 3I'”). SOX10 co-binds many loci with MITF

364 (Laurette et al., 2015), if SOX10 is absent from mutually-dependent peaks this may

365 explain the dependence of TFAP2 binding on MITF at these sites. At $\sim 40 \%$ (288/

366 717) of the mutually dependent peaks, the polycomb repressive histone mark

367 H3K27Me3 was significantly higher (including in the gene body) in MITF-KO cells 
but, unexpectedly, not in TFAP2-KO cells, even though MITF binding was lower in TFAP2-KO cells (illustrated in Fig 31', Supplemental Fig. 8E-G).

In summary, at about one third of MITF peaks that overlap TFAP2A peaks the MITF binding depends on TFAP2. Such TFAP2-dependent MITF peaks occur both at loci where nucleosome packing depends on TFAP2 (pioneer factor mode) and where it does not (non-pioneer factor mode). At a subset of TFAP2-dependent MITF peaks where TFAP2 acts in pioneer factor mode and characterized by the absence of SOXE binding site TFAP2A binding is, reciprocally, MITF-dependent.

At additional subsets of MITF peaks overlapping TFAP2A peaks, TFAP2 paralogs inhibit or have no effect on chromatin access for MITF

378 In Figure 2 we established that at some TFAP2A peaks, TFAP2 paralogs close chromatin, and presumably inhibit binding of transcription factors. Consistent with this prediction, among MITF peaks overlapping TFAP2A peaks, the height of $10 \%$ $(1,605)$ was higher in TFAP2-KO cells than in WT cells (Fig. 3A, E; Supplemental Fig. S9). At 58\% $(924 / 1,605)$ of these, the ATAC-seq signal was also significantly higher in TFAP2-KO cells versus in WT cells (violin plot, Fig. $\mathbf{3 H}, \mathbf{H}$ ', illustrated in Fig. 3H'). A unique set of transcription factor binding sites, including that for SP1, NFY, JUN and TFE3, were enriched among such elements (Fig. 3H'”'). Moreover, these elements were modestly associated with TFAP2-inhibited genes.

Of note, at the majority of MITF peaks that overlap TFAP2A peaks $(65 \%, 10,418$ / 15,752), the height was equivalent in TFAP2-KO and WT cells (Supplemental Fig. S9). Interestingly TFAP2-independent MITF peaks were not strongly enriched for the TFAP2 binding site (Supplemental Fig. S11A), implying that TFAP2 is attracted to many of these sites via other proteins rather than binding directly to the DNA. Such indirect binding may be less avid, as the average height TFAP2-independent MITF peaks was smaller than that of TFAP2-dependent MITF peaks (compare WT MITF signal in (Supplemental Fig. S9, compare cluster 1 and 4 in WT cells). As 
398 The delayed pigmentation in zebrafish tfap2a/tfap2e double mutants was consistent

399 with two mechanisms which are not exclusive of one another. In the first mechanism,

400 TFAP2 paralogs directly activate MITF expression, and thereby indirectly activate

401 expression of pigmentation genes. In the second mechanism, TFAP2 paralogs

402 directly activate expression of pigmentation genes. Supporting the first mechanism,

403 there is a pioneered TFAP2-activated enhancer in intron 2 of the MITF gene

404 (Supplemental Fig. S12), and MITF mRNA levels are about 40\% lower in TFAP2-

$405 \mathrm{KO}$ cells than in WT cells. However, the first mechanism predicts that loss of TFAP2

406 would most strongly diminish the expression of the most highly MITF-dependent

407 genes. However, many of the genes whose expression was most strongly reduced in

408 MITF-KO cells compared to in WT cells were completely TFAP2-independent, or

409 indeed were TFAP2-inhibited (Supplemental Table 4). To assess the second

410 mechanism, we identified the set of genes activated directly by MITF, defined as

411 MITF-activated genes associated with an MITF peak, and the set of genes directly

412 activated by TFAP2, defined as TFAP2-activated genes associated with an TFAP2-

413 activated enhancer (i.e., of pioneered or non-pioneered variety). Supporting the

414 second mechanism, genes activated directly both by TFAP2 and by MITF were

415 enriched for GO terms related to pigmentation (Fig. 4B), although genes related to

416 pigmentation were among those apparently directly regulated solely by MITF or

417 TFAP2 paralogs (Fig. 4C) (Baxter et al., 2019). We took a similar approach to

418 identify genes directly inhibited by TFAP2 and/or by MITF (Fig. 4D). Genes directly

419 inhibited by both were strongly enriched for $\mathrm{GO}$ terms related to cell adhesion and

420 cell migration (Fig. 4E). In summary, reduced expression of pigmentation genes and

421 elevated expression of invasion genes in TFAP2-KO cells compared to in WT cells is

422 largely explained by the direct activation and inhibition, respectively, of these

423 categories of genes by TFAP2 paralogs.

424 Considering the strong correlation of TFAP2-inhibited genes with cell migration we

425 performed in vitro scratch-recovery-assays and characterized the migrative capacity

426 of our TFAP2-KO cells. Unexpectedly, while WT SK-MEL-28 cells closed the wound

427 after 24 hours, both of our TFAP2-KO clones (clone 4.3 and clone 2.12) failed to

428 close the wound within that time (Supplemental Fig. 13D). This finding also

429 contrasts with the observation that the expression tfap2e correlates negatively with

430 the migratory capacity of zebrafish models of melanoma (Campbell et al., 2021), but 
431 it is consistent with the accumulation of melanocytes in the dorsum of zebrafish

432 tfap2a knockout embryos (Barrallo-Gimeno et al., 2004; Knight et al., 2004; Knight et

433 al., 2003) and tfap2a/ tfap2e double mutant embryos (Fig. 1, Supplemental Fig.S1).

434 Furthermore, these results are consistent our previous findings that knocking-down

435 MITF negatively influences cell migration and invasion (Dilshat et al., 2021). The

436 reduced migrative capacity of TFAP2-KO cells may be attributed to the strong up-

437 regulation of genes associated with cell adhesion in TFAP2-KO cells.

439 Finally, we considered how TFAP2 paralogs might regulate the phenotype in 440 melanoma cells. Advanced melanoma is characterized by lower levels of TFAP2A

441 than benign nevi (e.g., Huang et al., 1998), and low transcript levels of tfap2

442 paralogs are associated with an invasive phenotype in zebrafish melanoma

443 (Campbell et al., 2021). We examined the association of TFAP2-activated and

444 TFAP2-repressed genes (Supplemental Fig. 13A-C) with gene expression profiles

445 from melanoma tumors and melanoma cell lines with distinct phenotypes (Hoek et 446 al., 2008a; Hoek et al., 2006; Jonsson et al., 2010; Rambow et al., 2018; Tirosh et 447 al., 2016; Tsoi et al., 2018; Verfaillie et al., 2015). Enrichment analysis showed (Yu 448 et al., 2012) melanoma profiles previously found to be associated with high levels of 449 MITF activity were enriched for genes directly activated by TFAP2, including the 450 subset associated with TFAP2-dependent MITF peaks (Fig. 4F). Moreover, 451 melanoma profiles associated with low levels of MITF activity were enriched for 452 genes directly by TFAP2 (Fig. 4F).

\section{Discussion}

In this study, we confirm that Tfap2 paralogs are necessary for timely pigmentation in zebrafish embryos, as well as for normal levels of the expression of pigmentation genes in a melanoma cell line. We also describe how Tfap2 affects expression of such genes, and test the hypothesis that it makes chromatin more accessible to MITF, a transcription factor known to directly regulate the expression of pigmentation genes. The latter involved assessing the consequences of the loss of MITF alone, and that of both TFAP2A and TFAP2C, on global gene expression; on chromatin 
marks indicative of enhancers, promoters, and repressed chromatin; on nucleosome positioning; and on the binding of TFAP2A to chromatin in MITF mutants and that of MITF to chromatin in TFAP2A/TFAP2C double mutants. Integration of these datasets yielded a deeper understanding of the mechanisms whereby TFAP2 regulates gene expression than could be acquired by more traditional methods.

Integrating genomic data sets permitted us to identify genomic elements that were bound by TFAP2A in WT cells and that either lost or gained H3K27Ac signal in TFAP2-KO cells; we inferred that these elements were enhancers directly activated or inhibited by TFAP2. Of note, only a minority of TFAP2A peaks coincided with TFAP2-dependent enhancers. As expected by the Tfap2 as pioneer factor model, at a subset of TFAP2A-dependnet enhancers the ATAC-signal was TFAP2-activated. Interestingly, there were elements where the ATAC-seq signal was TFAP2 activated but the H3K27Ac signal independent and such elements were strongly associated with TFAP2-activated genes. It was also interesting that at a subset of TFAP2inhibited enhancers, loss of TFAP2 led to opening of the chromatin, implying that TFAP2 paralogs recruit distinct transcription factors, and that these in turn recruit either enzymes that open chromatin or enzymes that condense it, in locus-specific fashion. The latter is consistent with findings for FOXA1, which has been shown to recruit proteins that condense chromatin, like GRG3 (Sekiya and Zaret, 2007; Watts et al., 2011).

A second discovery from our analyses is that TFAP2 can activate enhancers in a non-pioneer factor mode. At a subset of TFAP2A peaks where the H3K27Ac signal was TFAP2-activated, the ATAC-seq signal was TFAP2-independent. Further evidence that such elements are TFAP2-dependent enhancers is that their average H3K4Me3 signal was also TFAP2-dependent. We infer that TFAP2 activates and inhibits these enhancers, but not as a pioneer factor. At such enhancers the continued presence of TFAP2 is necessary for continued acetylation of histone H3 lysine 27 (H3K27Ac), which fits with evidence that TFAP2 binds the histone acetyl transferase p300/CBP (Braganca et al., 2003) and inhibits the NURD histonedeacetylase complex (White et al., 2021). TFAP2 may attract other transcription factors without affecting nucleosome positioning; indeed, some TFAP2-dependent MITF peaks were found at Non-pioneered TFAP2- activated enhancers. The fact that 
the TFAP2 binding site is not strongly enriched at these Non-pioneered TFAP2activated enhancers may imply that TFAP2 binds these elements indirectly. Finally, we refer to these elements as non-pioneered TFAP2-activated enhancers because in the absence of TFAP2 their activity is lost but chromatin stays open. Our experimental design could not rule out the possibility that TFAP2 stably pioneered these elements such that chromatin remained open (but not active) after TFAP2 was removed. A precedent for this scenario is that at a subset of elements pioneered by PAX7, chromatin remains open after the removal of PAX7 (Mayran et al., 2018). However, the observation that TFAP2 site is less enriched compared to that of pioneered TFAP2-activated enhancers, and that the binding sites of pioneer factors FOS and JUN are enriched (Bejjani et al., 2019), supports the alternative model that such elements are simply pioneered by different transcription factors.

A third finding from this study is that at a subset of MITF/TFAP2A co-bound peaks, MITF binding was reduced in TFAP2-KO cells. A subset of such TFAP2-dependent MITF peaks were present at Pioneered TFAP2-activated enhancers, and at TFAP2independent NDRs (some of which were Non-pioneered TFAP2-activated enhancers, where the mechanism of recruitment of MITF is distinct). Thus, TFAP2 modulates MITF activity at certain loci by providing access to chromatin. Of note, at a subset of MITF/TFAP2A peaks, TFAP2 binding was lost in MITF-KO cells. There is precedent for reciprocal binding for pioneer factors, in the cases of both FOXA1 and steroid hormone receptors, at subsets of sites where they are co-bound (Swinstead et al., 2016a). Why are all MITF/TFAP2A peaks not mutually dependent? Notably, at many MITF/TFAP2A mutually dependent peaks, the repressive mark H3K27Me3 accumulated in MITF-KO cells. This is consistent with evidence a) that the SWI/SNF complex, which MITF probably recruits to such loci, competes for access to chromatin against the Polycomb repressor complex, which deposits H3K27Me3 (Wilson et al., 2010), and b) that the binding of pioneer factors is impeded by condensed H3K27me3-positive chromatin (Petruk et al., 2017; Wilson et al., 2010). SOX10, in MITF KO cells, but this is not the case for MITF/TFAP2A mutually dependent peaks. 
531 Finally, our results suggest a mechanism that could account for how TFAP2

532 promotes the pigmentation of embryonic melanophores, the expression of

533 pigmentation genes, and possibly the proliferation of melanoma cells. First, MITF

534 expression is lower in TFAP2-KO than WT cells. Second, because TFAP2 facilitates

535 binding of MITF to enhancers and promoters of genes that govern pigmentation, the

536 presence of TFAP2 leads to higher expression of those genes. Although the set of

537 MITF-activated but TFAP2-independent genes was not enriched for pigmentation

538 genes, it did include some such genes including MLANA, TYRP1 and PMEL.

539 Notably, despite lower MITF mRNA expression in TFAP2-KO cells, binding of MITF

540 was unchanged at such genes. This might explain why zebrafish embryonic

541 melanocytes become pigmented more slowly in tfap2a/tfap2e double mutants: the

542 number of melanophores in these mutants animals was reduced in embryos

543 depleted for tfap2a, singly or in combination with tfap2e, and the doubling time of

544 TFAP2-KO cells was longer than that of their WT counterparts. Also, MITF is known

545 to promote proliferation, but whether TFAP2 and MITF cooperate to promote

546 proliferation remains unclear. The expression of genes promoting cell migration and

547 invasion has been observed to be higher in cells with low versus high MITF activity

548 (Rambow et al., 2019). Indeed we show evidence suggesting that TFAP2 paralogs

549 directly suppress such genes, consistent with our previous findings (Campbell et al.,

550 2021). Thus, independent of its other activities as a transcription factor, TFAP2

551 determines which genes can be activated by MITF. In summary, MITF activity in

552 melanoma cells - and thus the phenotypes of these cells - depend in part on the

553 presence of transcription factors that give MITF access to specific regulatory

554 elements.

555

556 Materials and Methods

558 Zebrafish lines and maintenance

560 D. rerio were maintained in the University of lowa Animal Care Facility according to a 561 standard protocol (protocol no. 6011616). All zebrafish experiments were performed

562 in compliance with the ethical regulations of the Institutional Animal Care and Use

563 Committee at the University of lowa and in compliance with NIH guidelines. 
564 Zebrafish embryos were maintained at $28.5^{\circ} \mathrm{C}$ and staged by hours or days post-

565 fertilization (hpf or dpf).

\section{Generation of a zebrafish tfap2e loss-of-function allele}

To generate the tfap2e loss-of-function allele, we designed paired (e.g., left and right) zinc finger nucleases (ZFN) targeting exon 2 of the tfap2e locus resulting in non-homologous end-joining and disruption of the open reading frame for Tfap2e. Briefly, the online tool, ZiFiT (Sander et al., 2010), was used to identify an optimal ZFN target site [utilizing the CoDa approach (Sander et al., 2011). Once identified, a custom DNA fragment encoding the entire left or right zinc finger array (ZFA) along with flanking Xbal and BamHI restriction sites was synthesized (Integrated DNA Technologies, Coralville, IA). Subsequently, the ZFA fragment was subcloned into pMLM290 (Addgene, plasmid 21872), which includes a modified Fokl nuclease domain (Miller et al., 2007). Next, the fully assembled ZFN was PCR amplified, directionally cloned into pENTR-D/TOPO (ThermoFisher Scientific), and finally subcloned into pCS2+DEST using Gateway LR Clonase II enzyme mix (ThermoFisher Scientific). Once assembled, the final pCS2+ plasmids were sequence verified, linearized, mRNA synthesized in vitro (mMessage mMachine SP6 Kit, Ambion/ThermoFisher Scientific). Synthesized RNA was cleaned using the Qiagen RNeasy Kit (Qiagen) and both left and right ZFN components were coinjected into 1-cell stage zebrafish embryos. Following injections, embryos were initially screened via PCR and restriction enzyme digest to confirm editing at the target site. Upon confirmation, additional embryos from a similar clutch (F0's) were allowed to develop into adulthood, 'mosaics' identified and out-crossed, and a stable $\mathrm{F} 1$ generation isolated.

\section{Cell lines, reagents, and antibodies}

594 The cells referred to as WT throughout the document are the parent SK-MEL-28 (HTB-72) line. They and the derivative line, delta6-MITF knockout cells (referred to as MITF-KO cells in this work), were obtained from the laboratory of Dr. Eirikur 
supplemented with $10 \%$ FBS (Gibco \#10270106) at $5 \% \mathrm{CO}_{2}$ and $37^{\circ} \mathrm{C}$. Cells were tested for, and determined to be free of, mycoplasma. SK-MEL-28 cells harbor the BRAF $^{\mathrm{V} 600 \mathrm{E}}$ and $\mathrm{p} 53^{\mathrm{L} 145 \mathrm{R}}$ mutations (Leroy et al., 2014). The following primary antibodies and their respective dilutions were used in western blotting (WB) and CUT\&RUN experiments: anti-Tubulin (Sigma, \#T6199), 1:5000 (WB); anti-MITF (Sigma, \#HPA003259), 1:2000 (WB), 1:100 (CUT\&RUN); anti-TFAP2A (Abcam, ab108311), 1:5000 (WB), 1:200 (CUT\&RUN); anti-TFAP2C (Santa-Cruz \#SC-12762 X ), 1:1000 (WB); anti-H3K27Ac (EMD Millipore, \#07-360), 1:100 (CUT\&RUN); antiH3K4Me3 (EMD Millipore, \#05-745R), 1:100 (CUT\&RUN); H3K27Me3 (EMD Millipore, \#07-449), 1:100 (CUT\&RUN); Rabbit IgG (EMD Millipore,\#12-370), 1:100 (CUT\&RUN); Mouse IgG (EMD Millipore, \#12-371), 1:100 (CUT\&RUN). The following secondary antibodies and their respective dilutions were used: Anti-mouse $\lg \mathrm{G}(\mathrm{H}+\mathrm{L})$ DyLight 800 conjugate (Cell Signaling Technologies, \#5257), 1:20000; and anti-rabbit $\lg \mathrm{G}(\mathrm{H}+\mathrm{L})$ DyLight 680 conjugate Cell Signaling Technologies, \#5366), 1:100. Images were captured using an Odyssey CLx Imager (LICOR Biosciences).

\section{Purification of pA/G-MNase}

E.coli strain BL21-DE3 was transformed with plasmid DNA pAG-MNase-6xHis (Addgene, plasmid \#123461). Recombinant pAG-MNase was purified from cells grown in LB medium to OD600 0.6 at $37^{\circ} \mathrm{C}$. Cells were induced with $0.5 \mathrm{mM}$ IPTG and cultured for 16 hours at $20^{\circ} \mathrm{C}$. Cell pellets were homogenized in lysis buffer (10 $\mathrm{mM}$ Tris, pH 7.5, $300 \mathrm{mM} \mathrm{NaCl}, 10 \mathrm{mM}$ imidazole) containing lysozyme and protease inhibitors, then sonicated and the slurry was cleared by centrifugation (35K RPM, Ti70 rotor). The supernatant was subjected to IMAC chromatography (NI-NTA column) and to size-exclusion fractionation (Superdex 75) using a BioLogic DuoFlow QuadTec FPLC system (Bio-Rad). The purified pAG-MNase was concentrated by buffer exchange with ultrafiltration (Amicon Ultra-15, 10K). Finally, the purified pAGEDTA, and $50 \%$ glycerol), and stored at $-80^{\circ} \mathrm{C}$. 
632 TFAP2-KO clones were generated using the Alt-R CRISPR-Cas9 technology from

633 Integrated DNA Technologies (IDT). Briefly, crRNAs targeting exon 2 of TFAP2A and

634 TFAP2C were designed using the Cas9 guide RNA design checker tool (crRNA

635 sequences below). Equimolar concentrations of crRNA and tracrRNA (IDT,

636 \#1072532) were annealed to form gRNA complexes. The ribonucleoprotein (RNP)

637 complex was prepared by mixing gRNAs and Cas9 protein (IDT \#1081058). SK-

638 MEL-28 cells were transfected with constructs encoding components of RNP

639 complexes using the Lipofectamine CRISPRmax Cas9 transfection reagent

640 (ThermoFisher \#CMAX00015) following the manufacturer's protocol. Single-cell

641 colonies were screened by PCR and Sanger sequencing using primers flanking the

642 cut sites (primer sequences below). Mutant clones (clone 2.12 and clone 4.3) were

643 selected and further screened by western blotting, using anti-TFAP2A and anti-

644 TFAP2C antibodies. The control cell lines used in this study were generated

645 following this protocol but without adding gRNA duplexes.

crRNA

TFAP2A_ex2_gRNA1

TFAP2A_ex2_gRNA2

TFAP2C_ex2_gRNA1

TFAP2C_ex2_gRNA2

\section{Primers}

TFAP2A_geno_Fw

TFAP2A_geno_Rv

TFAP2C_geno_Fw

TFAP2C_geno_Rv
Sequence (5'-3')

CGTCACGACGGCACCAGCAAGTTTTAGAGCTATGCT

CTTACCTCACGCCATCGAGGGTTTTAGAGCTATGCT

CGCCACGACGGGAGCAGCAAGTTTTAGAGCTATGCT

CCACGACATGCCTCACCAGAGTTTTAGAGCTATGCT

Sequence (5'-3')

TCTCTTGTGCCCCСTCCATA

GCCCACCGACTGTATGTTCCA

CCGTGACCCCGATTTTGGAT

CGGCTTCACAGACATAGGCA
648

649

650

651

652

653

654

655

\section{SDS-PAGE and Western blotting}

TFAP2-KO and WT cells were washed in ice-cold PBS. RIPA buffer containing protease inhibitors (Roche, cOmplete Mini) was added and cells were lysed on ice for 20 minutes. Cell lysates were centrifuged at $14,000 \mathrm{~g}$ for 20 minutes and the quantity of protein in the supernatants was quantified using Bradford assays (Bio- 
Rad \#5000002). Laemmli sample buffer (Bio-Rad \#1610747, 5\% 2-mercaptoethanol) was added to $20 \mu \mathrm{g}$ protein and samples were boiled at $95^{\circ} \mathrm{C}$ for 5 minutes before being loaded onto a 10\% SDS-polyacrylamide gel (Bio-Rad \#4568034). Protein was transferred to polyvinylidene fluoride (PVDF) membranes (Thermo Scientific \#88520), which were incubated overnight with primary antibody. Membranes were washed 3 times with TBS-T and incubated with horseradish peroxidase-conjugated anti-rabbit or anti-mouse for 1 hour at room temperature, washed, and imaged using an Amersham Imager 600.

664

665

666

667

668

\section{ATAC-seq}

669

670

ATAC-seq was performed according to (Buenrostro et al., 2015; Liu et al., 2020) with minor alterations. Briefly, 70,000 TFAP2-KO cells (clone 2.12 and clone 4.3, four replicates each) and WT cells (four replicates) were lysed in ice-cold lysis buffer (10 mM Tris- $\mathrm{HCl}, \mathrm{pH}$ 7.4, 10 mM NaCl, 3 mM MgCl2, 0.1\% NP-40: Sigma).

674 Transposition was performed directly on nuclei using $25 \mu$ lagmentation reaction mix 675 (Tagment DNA Buffer \#15027866, Tagment DNA Enzyme \#15027865 from Illumina 676 Tagment DNA kit \#20034210). Tagged DNA was subjected to PCR amplification and library indexing, using the NEBNext High-Fidelity 2x PCR Master Mix (New England Biolabs \#M0451S) with Nextera DNA CD Indexes (Illumina \#20015882), according to the following program: $72{ }^{\circ} \mathrm{C}$ for 5 minutes; $98^{\circ} \mathrm{C}$ for 30 seconds; 12 cycles of $98{ }^{\circ} \mathrm{C}$ for 10 seconds, $63^{\circ} \mathrm{C}$ for 30 seconds, and $72{ }^{\circ} \mathrm{C}$ for 1 minute. The PCR product was purified with 1.8 times the volume of Ampure XP beads (Beckman Coulter \#A63881).

682 Library quality was assessed using a BioAnalyzer 2100 High Sensitivity DNA Chip 683 (Agilent Technologies). All DNA libraries that exhibited a nucleosome pattern were pooled and processed for $150 \mathrm{bp}$ paired-end sequencing. 
ATAC-seq was performed using 150 bp paired-end sequencing reads. Raw ATACseq reads were trimmed using Trim Galore Version 0.6.3 (Developed by Felix Krueger at the Babraham Institute) and aligned to human genome assembly hg19 (GRCh37) using Bowtie 2 (Langmead and Salzberg, 2012; Langmead et al., 2009) with default parameters. Sorting, removal of PCR duplicates, and identification of fragments shorter than $100 \mathrm{bp}$ as the nucleosome-depleted-regions (NDRs), was performed using BAM filter version 0.5.9. DeepTools version 3.3.0 (Ramírez et al., 2016) was used to check the reproducibility of the biological replicates and generate bigWig coverage files for visualization. Peaks were called using model-based analysis of ChIP-seq 2 (MACS2, version 2.1.1.20160309.6) (Zhang et al., 2008). NDRs for which accessibility differed between TFAP2-KO and WT cells were identified using DiffBind version 2.10 (Ross-Innes et al., 2012) with log2 fold-change threshold of $>1$ and a false discovery rate $(F D R)<0.05$. NDRs that are directly regulated by TFAP2 were identified by overlapping differentially accessible NDRs with anti-TFAP2A CUT\&RUN peaks; a 1-bp window was used to define overlap. Peaks were assigned to genes using GREAT with a peak-to-gene association rule of the nearest-gene-within-100 kb (McLean et al., 2010). Both the raw ATAC-seq files and processed sequencing data presented in this manuscript have been deposited in the Gene Expression Omnibus (GEO) repository (GSE number pending).

\section{CUT\&RUN}

CUT\&RUN sequencing was performed in TFAP2-KO cells (clone 2.12 and clone 4.3, two replicates each) and WT cells (two replicates) as previously described (Meers et al., 2019; Skene and Henikoff, 2017), but with minor modifications. Cells in logphase culture (approximately $80 \%$ confluent) were harvested by cell scraping, centrifuged at $600 \mathrm{~g}$ (Eppendorf, centrifuge 5424) and washed twice in calcium-free wash-buffer (20 mM HEPES, pH7.5, $150 \mathrm{mM} \mathrm{NaCl}, 0.5 \mathrm{mM}$ spermidine, and protease inhibitor cocktail cOmplete Mini, EDTA-free from Roche). Pre-activated concanavalin A-coated magnetic beads (Bangs Laboratories, Inc) were added to cell suspensions $\left(2 \times 10^{5}\right.$ cells) and incubated for 15 minutes at $4^{\circ} \mathrm{C}$. Antibody buffer (wash-buffer with 2mM EDTA and $0.05 \%$ digitonin) containing anti-TFAP2A, antiMITF, anti-H3K4Me3, anti-H3K27Me3, anti-H3K27Ac or Rabbit IgG was added and 
cells were incubated overnight at $4^{\circ} \mathrm{C}$. Cells were washed in dig-wash buffer (wash buffer containing $0.03 \%$ digitonin), and PAG-MNase was added at a concentration of $500 \mu \mathrm{g} / \mathrm{mL}$. The pAG-MNase reactions were quenched with 2X Stop Buffer (340mM $\mathrm{NaCl}, 20 \mathrm{mM}$ EDTA, 4mM EGTA, 0.05\% digitonin, $100 \mu \mathrm{g} / \mathrm{mL}$ RNAse A (10 mg/mL, Thermo Fisher Scientific \#EN0531), $50 \mu \mathrm{g} / \mathrm{mL}$ glycogen $(20 \mathrm{mg} / \mathrm{mL}$, Thermo Fisher Scientific \#R0561) and $2 \mathrm{pg} / \mathrm{mL}$ sonicated yeast spike-in control). Released DNA fragments were treated with $1 \mu \mathrm{L} / \mathrm{mL}$ phosphatase $\mathrm{K}(20 \mathrm{mg} / \mathrm{mL}$, Thermo Fisher Scientific \#25530049) for 1 hour at $50^{\circ} \mathrm{C}$ and purified by phenol/chloroform-extraction and ethanol-precipitation. Fragment sizes were analyzed using a 2100 Bioanalyzer (Agilent).

\section{CUT\&RUN library preparation and data analysis}

CUT\&RUN libraries were prepared using the KAPA Hyper Prep Kit (Roche). Quality control post-library amplification consisted of fragment analysis using the 2100 Bioanalyzer (Agilent). Libraries were pooled, brought to equimolar concentrations, and sequenced with 150 bp paired-end reads on an Illumina HiSeq X platform (Novogene, Sacramento, CA). For quality control, paired-end FASTQ files were processed using FastQC (Babraham Bioinformatics). Reads were trimmed using Trim Galore Version 0.6.3 (Developed by Felix Krueger at the Babraham Institute) and then mapped against the hg19 genome assembly using Bowtie2 version 2.1.0 (Langmead and Salzberg, 2012; Langmead et al., 2009). The mapping parameters and peak calling of MACS2 peaks (Zhang et al., 2008) were performed as previously described (Meers et al., 2019; Skene and Henikoff, 2017) against their matching control IgG samples. Differential analysis of H3K27Ac and of $\mathrm{H} 3 \mathrm{~K} 27 \mathrm{Me} 3$ signal in WT and TFAP2-KO cells was preformed using MACS2 with a Log2 fold-change threshold of 1 , and $p$-value $<1 \times 10^{-5}$. Differential H3K4Me3, MITF and TFAP2A signal in WT, TFAP2-KO and when mentioned MITF-KO cells was determined using DiffBind version 2.10.0 (Ross-Innes et al., 2012) with a Log2 fold-change threshold of 1 , and FDR $<0.05$. DeepTools version 3.3.0 was used to check the reproducibility of the biological replicates, to generate bigwig normalized (RPKM) coverage files for visualization and to plot average CUT\&RUN-seq and ATAC-seq profiles (-plotProfile) and generate heatmaps (-plotHeatmap) of normalized reads (Ramírez et al., 2016). MultiBigwigSummary was used to extract read counts (-outRawCounts) (Ramírez et 
al., 2016) and Prism was used to generate Violin and Box plots. Peaks were assigned to genes using GREAT with a peak-to-gene association rule of the nearestgene-within-100 kb (McLean et al., 2010)

\section{RNA-seq}

Four replicate RNA-seq experiments were performed on TFAP2-KO cells (clone 2.12 and clone 4.3) and WT cells. Total RNA was extracted by direct cell lysis using the RNeasy Plus Mini Kit with QiaShredder (Qiagen \#47134). RNA samples with an RNA integrity number (RIN) above nine were used for library generation and 150 bp paired-end sequencing on the Illumina HiSeq2500 platform (Novogene, Sacramento, CA). FASTQ sequence files were processed using FastQC (Babraham Bioinformatics) for quality control, and reads were trimmed using Trim Galore Version 0.6.3 (Developed by Felix Krueger at the Babraham Institute) and subsequently aligned to human genome assembly hg19 (GRCh37) using STAR (Dobin et al., 2013). The output of the --quantMode GeneCounts function of STAR was used for the calculation of differential transcript expression using DESeq2 (Love et al., 2014).The rlog function was used to generate log2-transformed normalized counts. Adjusted p-value $<0.05$ was used as the threshold for statistically significant differences. Functional enrichment analyses was performed using PANTHER (Mi et al., 2021). A full list of genes differentially expressed between TFAP2-KO and WT cells is provided in Supplemental Table 1-3.

\section{Motif analyses}

Both de novo and known motifs were identified within 200 bp of TFAP2-activated and TFAP2-inhibited enhancer and promoter peak summits using HOMER (findMotifsGenome).

\section{Statistical analysis}


790 Fisher's Exact Test was used to assess TFAP2-regulated elements (enhancers and

791 promoters) with TFAP2-regulated gene expression. Data processing and analysis

792 was performed in $\mathrm{R}$ and the code can be found at

793 https://GitHub.com/ahelv/Differential Expression. GraphPad-Prism was used to

794 perform Students t-test as indicated in the figure legends

795

796

\section{Wound scratch assay}

797

798 A total of $500 \mathrm{~K}$ cells were seeded per well of 6-well plate (Thermo Scientific, \#

799 1483210) to reach confluent monolayer. Cells were incubated in serum free media

800 for 6 hours before wounding with a $200 \mu \mathrm{L}$ pipette tip. Scratches were manually

801 imaged on an inverted light microscope (Leica \#10445930) every 6 hours over a 24-

802 hour time period. The distance of scratch closure between WT and TFAP2-KO cells

803 were analyzed with Image J software.

804

805 Acknowledgements

806 This work was supported by grants from the National Institutes of Health (NIH) to

807 RAC (R01-AR062457), and a postdoctoral fellowship from the American Association

808 for Anatomy (CK). The ES laboratory is supported by grants 207067 and 217768

809 from the Research Fund of Iceland.

810

\section{Author Contributions}

812

813 Conceptualization: CK, RAC.

814 Formal analysis: CK, RD.

815 Funding acquisition: CK, ES, RAC.

816 Investigation: CK, RAC.

817 Methodology: CK, RD, HS, EVO, AH, GB.

818 Statistical analysis: $\mathrm{CK}, \mathrm{AH}$.

819 Supervision: ES, RAC.

820 Writing - original draft: CK, RAC. 


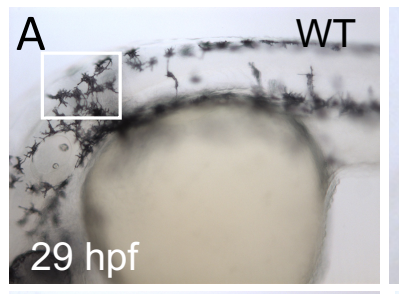

B

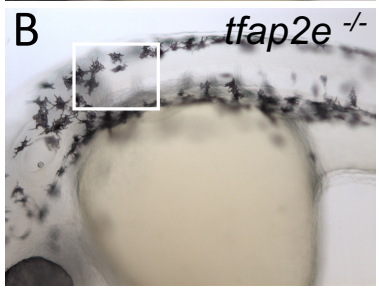

C

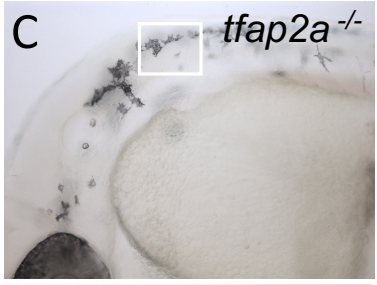

$\mathrm{D}$

$$
\text { tfap2a } a^{-1}
$$
tfap2e $e^{-1}$

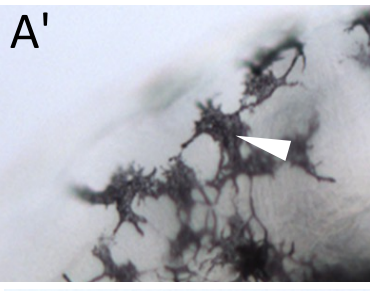

$\mathrm{B}^{\prime}$

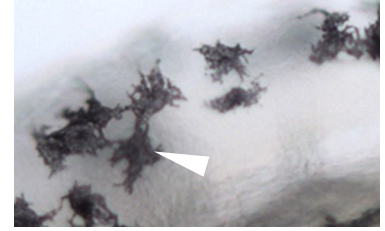

$C^{\prime}$

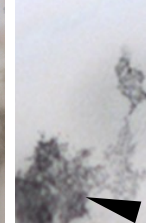

$D^{\prime}$
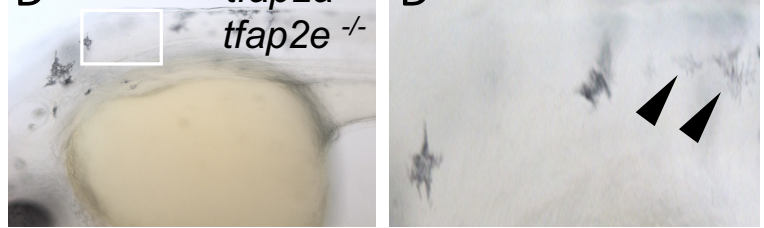

822 
bioRxiv preprint doi: https://doi.org/10.1101/2021.11.23.469757; this version posted November 30, 2021. The copyright holder for this

preprint (which was not certified by peer review) is the author/funder, who has granted bioRxiv a license to display the preprint in perpetuity. It is made available under aCC-BY-NC-ND 4.0 International license.

\section{A}

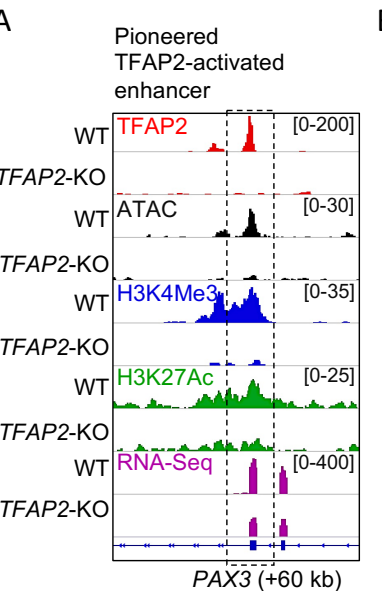

E

$$
\begin{aligned}
& \text { Pioneered } \\
& \text { TFAP2-activated } \\
& \text { enhancers }
\end{aligned}
$$

क़ $(2,002$ peaks $)$

ఖ $8{ }^{\mathrm{N}} \mathrm{H} 3 \mathrm{~K} 27 \mathrm{Ac}$

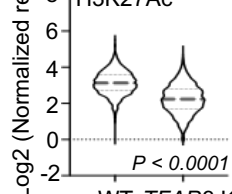

$\mathrm{E}^{\prime}$

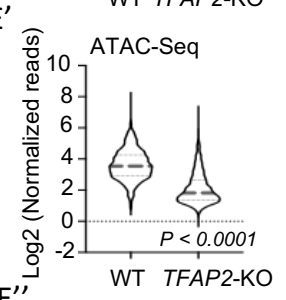

$E^{\prime \prime}$

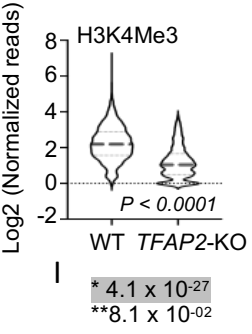

B

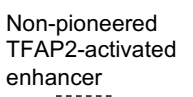

enhancer

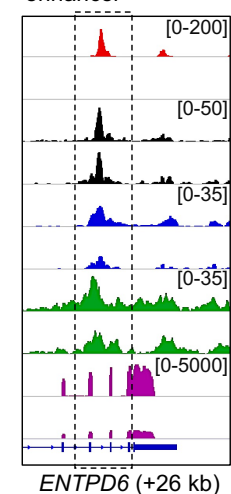

$\mathrm{F}$

Non-pioneered

TFAP2-activated

enhancers

(1,836 peaks)

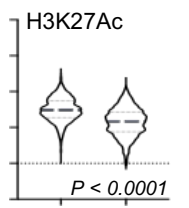

$\mathrm{F}^{\prime}$

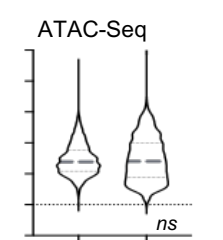

$F^{\prime \prime}$

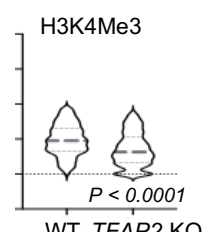

WT TFAP2-KO

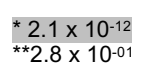

C Pioneered

TFAP2-inhibited

enhancer

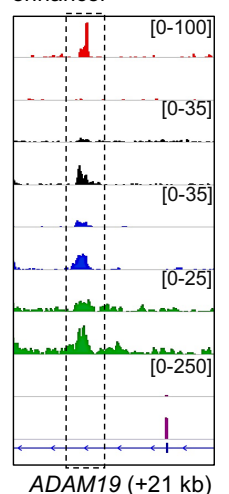

$\mathrm{G}$

Pioneered

TFAP2-inhibited

enhancers

(864 peaks)

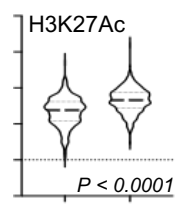

$\mathrm{G}^{\prime}$

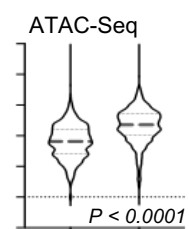

G"

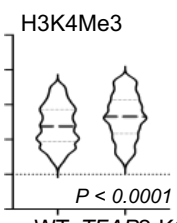

K

* ns

${ }^{* *} 9.4 \times 10^{-05}$
D Non-pioneered

TFAP2-inhibted

enhancer

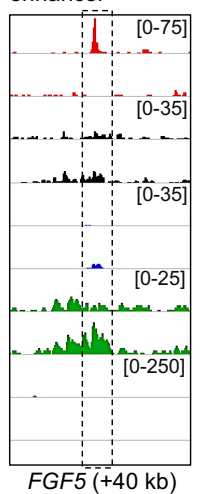

$\mathrm{H}$

Non-pioneered

enhancers

(440 peaks)

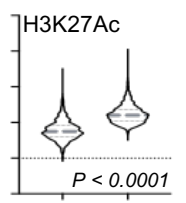

$\mathrm{H}^{\prime}$

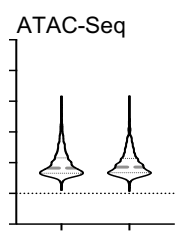

$\mathrm{H}^{\prime \prime}$

WT TFAP2-KO

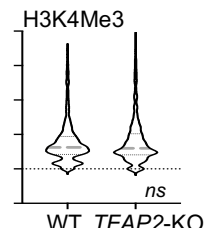

$\mathrm{L}$

${ }^{*}$ ns
Fig. 2

* $p$-val. enhancer association with TFAP2-activated genes ${ }^{* *} \mathrm{p}$-val. enhancer association with TFAP2-inhibited genes

M

Pioneered TFAP2-

activated enhancers

\begin{tabular}{clll}
\hline Motif & TF & Rank & P-value \\
\hline TCMCTCACC TFAP2 & 1,2 & $1 \times 10-600$
\end{tabular}

$\begin{array}{lll}\text { GCCCACCAAA EBF } & 3 & 1 \times 10^{-51}\end{array}$

CCTITTCG SOXE $4,6 \quad 1 \times 10^{-36}$

AACCACGAA RUNX $7 \quad 1 \times 10^{-31}$

GCATCTIAC MITF $28 \quad 1 \times 10^{-12}$
N

Non-pioneered TFAP2

activated enhancers

\begin{tabular}{clll}
\hline Motif & TF & Rank & P-value \\
\hline CHCCAT TFAP2 & 1 & $1 \times 10^{-280}$
\end{tabular}

CFAAGICITC FRA1 $2 \quad 1 \times 10^{-46}$

TAACCACA RUNX $4 \quad 1 \times 10^{-27}$

CAAGGATCC TEAD $6 \quad 1 \times 10^{-24}$

AAGCCTCT ZFX $7 \quad 1 \times 10^{-22}$
O

Preered TFAP2

\begin{tabular}{clll}
\hline Motif & TF & Rank & P-value \\
\hline CCTACCCAT TFAP2 & 1,2 & $1 \times 10^{-63}$
\end{tabular}

IF]TIITC RUNX $3 \quad 1 \times 10^{-37}$

ECCTITG CST6 $4 \quad 1 \times 10^{-21}$

ATCCCTGG ETS1 $6 \quad 1 \times 10^{-14}$

CCTCATCG CTCF $12 \quad 1 \times 10^{-12}$ 
bioRxiv preprint doi: https://doi.org/10.1101/2021.11.23.469757; this version posted November 30, 2021. The copyright holder for this

preprint (which was not certified by peer review) is the author/funder, who has granted bioRxiv a license to display the preprint in perpetuity. It is made available under aCC-BY-NC-ND 4.0 International license.

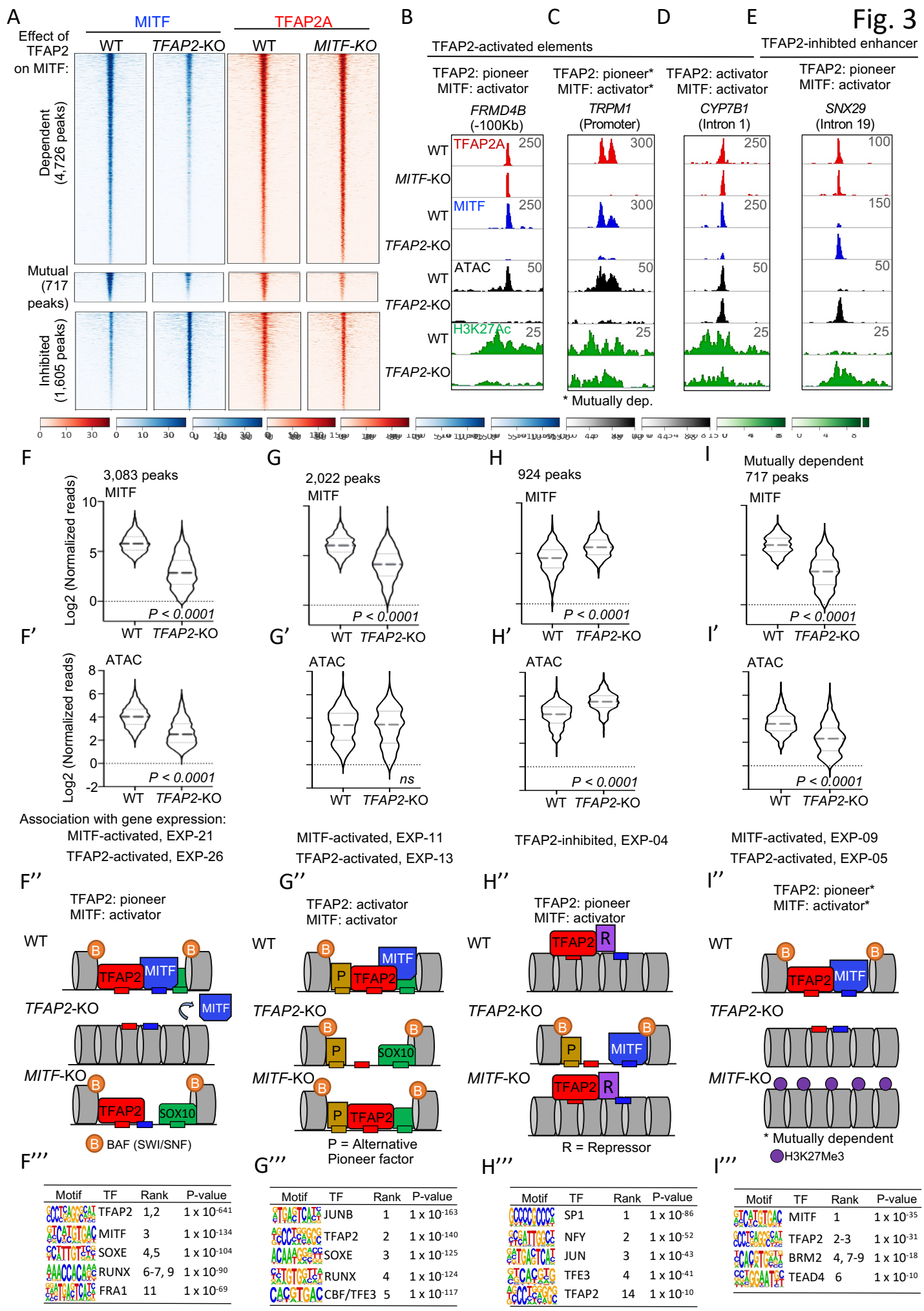

F

tion with gene expression MITF-activated, EXP-21

TFAP2: pioneer

TT

AACCCCGACONONX 6-7,9 $1 \times 10^{-90}$

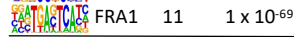

$G^{\prime \prime}$
B

\section{C}

D

E

Fig. 3

2: activator

pioneer

(Intron 1)

SNX29
(Intron 19)

।

924 peak

ATAC

$$
\text { WT TFAP2-KO }
$$

TF-activated, EXP-09 TFAP2-activated, EXP-05 TFAP2-activated, EXP-13

TFAP2-KO 
bioRxiv preprint doi: https://doi.org/10.1101/2021.11.23.469757; this version posted November 30, 2021. The copyright holder for this preprint (which was not certified by peer review) is the author/funder, who has granted bioRxiv a license to display the preprint in perpetuity. It is made available under aCC-BY-NC-ND 4.0 International license.

A

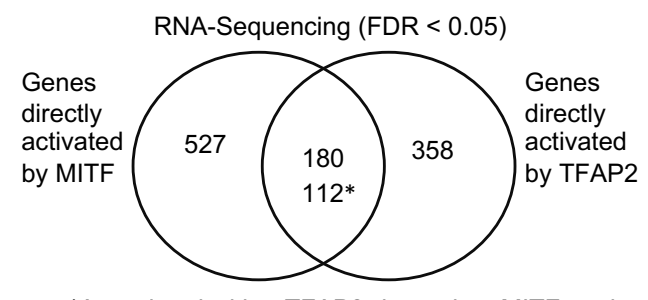

${ }^{*}$ Associated with a TFAP2-dependent MITF peak

B

MITF and TFAP2-activated genes (Top 5 hits)

\begin{tabular}{llll}
\hline GO biological process & Enrichment & $\begin{array}{l}\text { No. of } \\
\text { genes }\end{array}$ & FDR \\
\hline Pigmentation & 16.75 & 11 & $2.79 \times 10^{-06}$ \\
Pigment differentiation & 28.02 & 6 & $9.79 \times 10^{-04}$ \\
Cell differentiation & 44 & 77 & $8.7 \times 10^{-03}$ \\
Cellular pigmentation & 13.49 & 5 & $4.68 \times 10^{-02}$ \\
Receptor signaling & 3.51 & 15 & $3.78 \times 10^{-02}$ \\
\hline
\end{tabular}

C

Pigmentation related genes directly activated by:

$\begin{array}{ll}\text { MITF } & \text { MITF and TFAP2 } \\ \text { DKC1 } & \text { RAB1A } \\ \text { MLANA } & \text { RAB32 } \\ \text { EIF3B } & \text { HPS3 } \\ \text { KBTBD8 } & \text { CLCN7 } \\ \text { RAB27A } & \text { CTNNB1 } \\ \text { STX3 } & \text { CTSD } \\ \text { AFG3L2 } & \text { DCT } \\ \text { BNC2 } & \text { ETS1 } \\ \text { CCT2 } & \text { GPR143 } \\ \text { DTNBP1 } & \text { MAFB } \\ \text { IRF4 } & \text { MFSD12 } \\ \text { MLPH } & \text { MITF } \\ \text { MYO10 } & \text { MREG } \\ \text { OSTM1 } & \text { NR4A3 } \\ \text { PARD3 } & \text { OCA2 } \\ \text { PMEL } & \text { PEPD } \\ \text { PPARGC1A } & \text { RAB38 } \\ \text { PSEN2 } & \text { SH3BP4 } \\ \text { RPS14 } & \text { SH3PXD2A } \\ \text { RPS7 } & \text { SNAI2 } \\ \text { SCUBE2 } & \text { TRPM1 } \\ \text { SLC45A2 } & \text { VDR } \\ \text { STXBP1 } & \\ \text { SZT2 } & \\ \text { TYRP1 } & \\ \text { USF2 } & \\ \text { VPS18 } & \\ & \end{array}$

828
IGFBP7

KCTD15

ABCA12

CHD7

EDNRB

EN1

HIPK2

HTRA

MCM4

PARN

PAX3

$R A D 21$

SDC2

$S L C 2 A 11$

SOX9

SYTL2

TFAP2C

ALCAM

BLM

DISC1

ERBB3

FANCC

LYST

MIB1

NBN

PMCH

$P R K D C$
D

Fig. 4

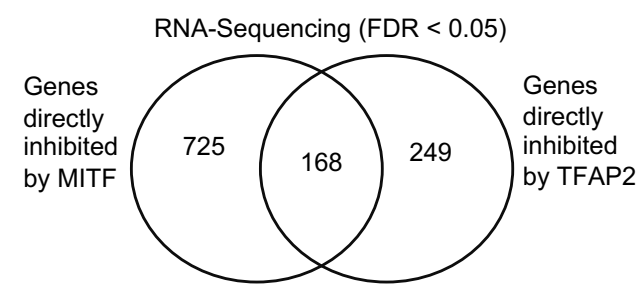

E

\begin{tabular}{llll}
\multicolumn{4}{l}{ MITF and TFAP2-inhibited genes (Top 5 hits) } \\
\hline $\begin{array}{l}\text { GO biological } \\
\text { process }\end{array}$ & Enrichment & $\begin{array}{l}\text { No. of } \\
\text { genes }\end{array}$ & FDR \\
\hline $\begin{array}{l}\text { Vasculature } \\
\text { development }\end{array}$ & 6.8 & 32 & $1.8 \times 10^{-12}$ \\
$\begin{array}{l}\text { Tube development } \\
\text { Cell motility }\end{array}$ & 5.13 & 36 & $1.4 \times 10^{-10}$ \\
Cell migration & 4.81 & 34 & $2.2 \times 10^{-08}$ \\
Cell adhesion & 3.51 & 31 & $3.7 \times 10^{-07}$ \\
\hline
\end{tabular}

F

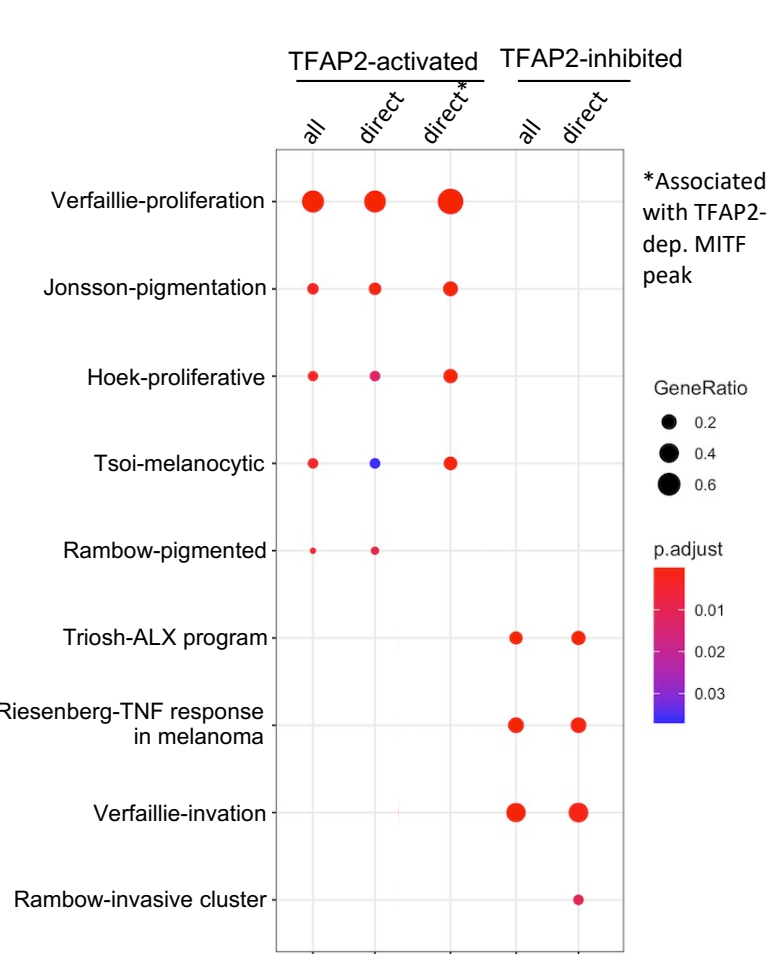


Figure legends

834 Figure 1: Stable KO of tfap2e has no effect on pigmentation in WT embryos but

mutants. (A-D) Lateral views of head and trunk of live embryos at $29 \mathrm{hpf}$, anterior to the left and dorsal to the top. (A-A') A WT (sibling) embryo with normal melanocytes

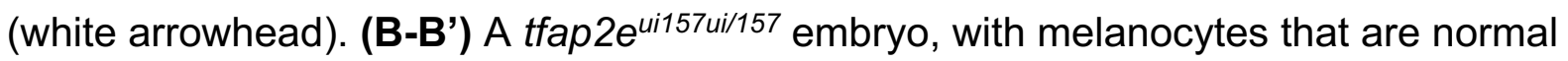
in terms of number, differentiation and pigmentation (white arrowhead). (C-C') A tfap2a $a^{\text {low/low }}$ homozygous mutant embryo, with fewer melanocytes than its tfap2 $e^{157 / 157}$ and WT sibling embryos. (D-D') A tfap2alow/low; tfap2e $e^{157 / 157}$ doublemutant embryo, with fewer melanin-producing melanophores than its tfap2alow/low sibling. (E) Box plot illustrating the number of pigmented melanocytes in the dorsum of WT (+/+), tfap2a mutant, tfap2e mutant, and double tfap2a; tfpa2e mutant embryos at $36 \mathrm{hpf}$. Center line, mean; box limits, upper and lower quartiles; whiskers, minimum and maximum values; black dots, number of melanocytes per

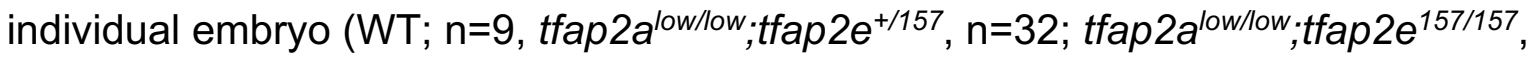
$n=10)$. P-value according to the Student's t-test.

Figure 2: TFAP2 paralogs facilitate gene expression by opening and anti-H3K4Me3 CUT\&RUN-seq (blue), anti-H3K27Ac CUT\&RUN-seq (green) and RNA-seq (magenta) datasets at (A) a pioneered TFAP2-activated enhancer at the PAX3 (+60 kb) locus (B) a non-pioneered TFAP2A-activated enhancer at the ENTPD6 (+26kb) locus (C) a pioneered TFAP2-inhibited enhancer at the ADAM19 $(+21 \mathrm{~kb})$ locus and (D) a non-pioneered TFAP2A-inhibited enhancer at the FGF5 (+40kb) locus. Genotypes as labeled; y-axes are grouped scaled per dataset. (E-E') Violin plots representing (E) anti-H3K27Ac (two independent replicates) (E') ATACseq (four independent replicates) and (E') anti-H3K4Me3 (two independent replicates) normalized reads at pioneered TFAP2-activated enhancers. (F-F') Violin

862 plots representing (F) anti-H3K27Ac (F') ATAC-seq and (E') anti-H3K4Me3 
representing (G) anti-H3K27Ac (G') ATAC-seq and (G') anti-H3K4Me3 normalized reads at pioneered TFAP2-inhibited enhancers. (H-H') Violin plots representing $\mathbf{( H )}$ anti-H3K27Ac (H') ATAC-seq and (H') anti-H3K4Me3 normalized reads at nonpioneered TFAP2-inhibited enhancers. P-values shown were determined by Students t-test. (I-L) Hypergeometric analysis of TFAP2 regulated enhancers at TFAP2-activated $\left({ }^{*}\right)$ and TFAP2-inhibited $\left({ }^{* *}\right)$ genes in TFAP2-KO cells (FDR $<0.05$, $|\log 2 \mathrm{FC}|>1)$. (M-O) Enrichment of transcription factor motifs at (M) pioneered TFAP2-activated enhancers, at (N) non-pioneered TFAP2-activated enhancers and at $(\mathrm{O})$ pioneered TFAP2-inhibited enhancers as determined using HOMER motif analysis. $P$ values were calculated using ZOOPS scoring (zero or one occurrence per sequence) coupled with hypergeometric enrichment analysis. TF; transcription factor.

Figure 3: TFAP2 paralogs facilitate chromatin access by MITF. (A) Density heatmaps of anti-MITF CUT\&RUN-seq in SK-MEL-28 and TFAP2-KO cells, and antiTFAP2 CUT\&RUN-seq in SK-MEL-28 and MITF-KO cells at TFAP2-dependent MITF peaks (top), mutually dependent peaks (center) and TFAP2-inhibited MITF peaks (bottom). Number of peaks in each group as labelled. Regions shown are $+/-3 \mathrm{~kb}$ browser (GRCH37/hg19), showing anti-TFAP2A (red) CUT\&RUN-seq in SK-MEL-28 and MITF-KO cells, and anti-MITF (blue) CUT\&RUN-seq, ATAC-seq (black) and anti-H3K27Ac (green) CUT\&RUN-seq profiles in SK-MEL-28 and TFAP2-KO cells. Examples of MITF binding at (B-D) TFAP2-activated and (E) TFAP2-inhibted regulatory elements. Genotypes as labeled; y-axes are grouped scaled per dataset. (F-F') Violin plot representing (F) anti-MITF CUT\&RUN-seq (two independent replicates) and (F') ATAC-seq (four independent replicates) at 3,083 pioneered TFAP2-activated MITF peaks. (G-G') Violin plot representing (G) anti-MITF CUT\&RUN-seq and (G') ATAC-seq at 2,022 non-pioneered TFAP2-activated MITF peaks. (H-H') Violin plot representing (H) anti-MITF CUT\&RUN-seq and (H') ATACseq at 924 pioneered TFAP2-inhibited MITF peaks and (I-I') Violin plot representing

894 (I) anti-MITF CUT\&RUN-seq and (I') ATAC-seq at 717 mutually dependent peaks. P895 value according to Students t-test, ns; not statistically significant, normalized reads 896 RPKM. Association with gene expression; hypergeometric analysis of TFAP2897 depednent and TFAP2-inhibited MITF peaks are shown at TFAP2-activated and 
MITF-activated genes (FDR < 0.05, log2FC > |1|). (F''- I') Schematic representation of TFAP2-dependent and TFAP2-inhibited MITF peaks as labelled; B; BAF complex (SWI/SNF), P; alternative pioneer factor. R; repressor protein. Transcription factor binding sites indicated by small rectangles, TFAP2 (red), MITF (blue) and alternative pioneer factor (yellow), example activator SOX10 (green) . (F'"' - l'”') Enrichment of transcription factor motifs using HOMER at $\left(F^{\prime \prime \prime}\right)$ pioneered TFAP2-dependent MITF peaks, (G'”) non-pioneered TFAP2-dependent MITF peaks, (H'”) TFAP2-inhibited MITF peaks and (I'") mutually dependent peaks. P values were calculated using ZOOPS scoring (zero or one occurrence per sequence) coupled with hypergeometric enrichment analysis. TF; transcription factor.

\section{Figure 4: TFAP2 and MITF co-regulate pigmentation and cell differentiation} genes in SK-MEL-28 cell lines. (A) Venn diagram representing directly MITF activated genes (MITF peaks within 100Kb of a TSS), based on RNA-seq, in MITFKO verses WT cells (FDR < 0.05) and genes directly activated by TFAP2 (TFAP2activated enhancers within $100 \mathrm{~Kb}$ of a TSS), based on RNA-seq, with TFAP2activated enhancers, in TFAP2-KO verses WT cells (FDR < 0.05). The number of overlapping genes with TFAP2-dependent MITF peaks are also shown $\left(^{*}\right)$. (B) Gene ontology (GO) biological process analysis (Top 5 hits) that are enriched among MITF- and TFAP2-activated genes. (C) A curated list of pigment-associated genes (Baxter et al., 2009) was intersected with directly MITF-activated, directly MITF/TFAP2-activated, and TFAP2-acitvated genes and represented by gene list.

(D) Venn diagram representing directly MITF inhibited genes, based on RNA-seq, in MITF-KO verses WT cells (FDR < 0.05) and genes directly inhibited by TFAP2, based on RNA-seq, with TFAP2-inhibited enhancers, in TFAP2-KO verses WT cells $($ FDR < 0.05).

(E) Gene ontology (GO) biological process analysis (Top 5 hits) that are enriched among MITF- and TFAP2-inhibited genes. GO analysis was performed using PANTHER. (F) Dot plot of enrichment analysis showing the enrichment of gene signatures from the literature in directly TFAP2-activated and TFAP2-inhibted genes, based on RNA-seq, in TFAP2-KO and SK-MEL-28 cells. $\mathrm{P}$ value is red lowest to blue highest; gene ratio is the ratio between genes and all genes in the GO category. Analysis of directly TFAP2-activated genes associated with TFAP2dependent MITF peaks are shown $\left(^{*}\right)$. 
932 Figure S1: tfap2e mutant zebrafish do not display a melanocyte phenotype whereas tfap2a/e double mutant zebrafish display a significant reduction in melanocyte number. (A) A 157 base pair mutation at the end of tfap2e exon 2 disrupts splicing and results in a premature stop codon. (B) PCR using primers in

936 tfap2e exon 2 and intron 2 (e2-i2) amplifies a band of the expected 450 base pair 937 size in tfap2e mutants but not wildtype (WT), whereas primers in exon 2 and exon 3 938 (e2-e3) amplify only in wildtype. NTC: not template control. (C) qRT-PCR analysis of 939 tfap2e expression shows that the transcript is strongly decreased in tfap2e-/-

940 mutants, consistent with nonsense-mediated decay (Student's t-test, ${ }^{* * *} p<0.0001$ ). (D-E) tfap2e mutant zebrafish at $36 \mathrm{hpf}$, tfap2e+/- (D) and tfap2e-/- (E) are

942 phenotypically indistinguishable. (F) Histogram illustrating the number of pigmented

943 melanocytes in the dorsum of tfap2e+/- and tfap2e-/- mutant zebrafish embryos,

944 (G-H) Zebrafish embryos from a tfap2a+/-;ffap2e+/- incross at 48 hpf. (G) A wildtype 945 embryo shows normal melanocyte patterning. (H) A tfap2a-/- mutant embryo has 946 fewer and paler embryonic melanocytes than wildtype. (I) tfap2a-/-;tfap2e+/- and (J)

947 tfap2a-/- ;ffap2e-/- appear phenotypically indistinguishable from tfap2a-/- at 48 hpf.

949 Figure S2: TFAP2 binds to open and closed chromatin. (A) Screenshot of IGV

950 genome browser (GRCH37/hg19), visualizing anti-TFAP2A and IgG CUT\&RUN-seq 951 profiles. Peaks were called using MACS2 software (two independent replicates) and 952 are illustrated by blue bars under the anti-TFAP2A track. (B) Density heatmap 953 centred on the 36,867 TFAP2A peaks identified by anti-TFAP2A CUT\&RUN in WT

954 SK-MEL-28 cells. Regions shown are $+/-3$ kb from peak center, Peaks were grouped by distance to an annotated transcriptional start site. Promoter peaks +/$3 \mathrm{~kb}$ from a TSS and enhancers $>3 \mathrm{~kb}$ from an TSS. Anti-TFAP2A, anti-H3K4Me3 and anti-H3K27Ac CUT\&RUN-seq, and ATAC-Seq profiles are shown. Normalized reads (RPKM). (C) Histogram representing H3K27Ac signal, binned from low-high read-depth on the $\mathrm{x}$-axis and percentage of TFAP2A promoter peaks (black) and

960 TFAP2A enhancer peaks (red) on the y-axis. (D) Violin plots illustrating TFAP2A and 961 IgG normalized reads (RPKM) at nucleosome depleted regions (ATAC-peaks) and at 962 nucleosome occupied DNA (no ATAC-peak) (E) Density heatmap representing 963 TFAP2A CUT\&RUN and ATAC-seq profiles at TFAP2A peaks that overlap 964 nucleosome depleted regions (ATAC-peaks) and at nucleosome bound DNA (no 965 ATAC-peak), the number of TFAP2A peaks in each group are as labeled. 
967 Figure S3: Example loci of TFAP2A peaks at open and closed chromatin. (A-B)

968 IGV screenshots of TFAP2A peaks that (A) closed chromatin and (B) open

969 chromatin, based on ATAC-seq, in SK-MEL-28 cells. Genes names and distance to

970 a transcriptional start site as labeled. (C) HOMER motif analysis at TFAP2A peaks at

971 closed chromatin and (D) at open chromatin. TF, transcription factors; the top

972 ranking transcription factor motif is shown, with P-values calculated with HOMER-

973 based hypergeometric enrichment analysis.

974

975 Figure S4: Generation of TFAP2A; TFAP2C double mutant SK-MEL-28 cell

976 lines. (A) RNA-seq showing transcript counts of TFAP2 paralogs in SK-MEL-28

977 cells. Transcript counts for WT cells $(n=4)$ and two TFAP2A;TFAP2C double

978 knockout clones (4 replicates each) are shown. The expression of WT and mutant

979 alleles of TFAP2A is comparable between cell lines whereas mutant alleles of

980 TFAP2C are strongly reduced. (B) Two guide RNAs (crRNAs) each were designed

981 to target exon 2 of TFAP2A and TFAP2C (yellow boxes). (C) A 401 base pair

982 inversion and a 452 base pair deletion at exon 2 of TFAP2A and TFAP2C,

983 respectively, was identified in clone 4.3. TFAP2A and TFAP2C mutant alleles

984 resulted in a frame-shift and premature stop codon in alleles of both genes. (D) A

985405 base pair deletion at exon 2 of TFAP2A resulted in a frame-shift and premature

986 stop codon in clone 2.12. A 455 base pair deletion, and a 70 base pair insertion, 1

987 base pair deletion (Indel) was identified in exon 2 of TFAP2C. Such mutations

988 resulted in a frame-shift and premature stop codon. Additional permutations were not

989 identified at exon 2 of TFAP2A or TFAP2C in clone 4.3 or clone 2.12 cells. Inv;

990 inversion, Del; deletion. (E) Western blot analysis confirming loss of TFAP2A and

991 TFAP2C immunoactivity in clone 4.3 and clone 2.12 cell lines.

992

993 Figure S5: TFAP2 paralogs activate and inhibit gene expression directly.

994 (A) Volcano plot illustrating differential gene expression as determined by RNA 995 sequencing of TFAP2-KO cells (two independent clones (2.12 and 4.3); four

996 replicates each) versus WT cells (SK-MEL-28; four replicates). Log2 fold change

997 (FC) of mean transcript levels on the x-axis and log2 p-value on the y-axis. Red dots

998 represent direct target genes of TFAP2A that are differentially expressed (FDR <

$9990.05, \log 2 \mathrm{FC}>|1|)$, as determined by anti-TFAP2 CUT\&RUN in SK-MEL-28 cells. 
1000 Gray dots represent genes that are differentially expressed but not bound by TFAP2.

1001 The number and percentage of genes that are regulated by TFAP2 are specified.

1002 Directly TFAP2A-dependent genes were identified based on the gene-association

1003 rule (single nearest gene within $100 \mathrm{~kb}$ of a transcription start site).

1004

1005 Figure S6: TFAP2 paralogs directly activate and inhibit promoters as pioneer

1006 factors. (A-D) Screenshot of IGV genome browser (GRCH37/hg19), visualizing anti-

1007 TFAP2A CUT\&RUN-seq (red), ATAC-seq (black), anti-H3K4Me3 CUT\&RUN-seq

1008 (turquoise), anti-H3K27Ac CUT\&RUN-seq (green) and RNA-seq (magenta) datasets

1009 at (A-B) TFAP2-activated promoters and (C-D) TFAP2-inhibited promoters.

1010 Genotypes as labeled; y-axes are grouped scaled per dataset. (E-E') Violin plots

1011 representing (E) anti-H3K27Ac (two independent replicates) (E') ATAC-seq (four

1012 independent replicates) and (E') anti-H3K4Me3 (two independent replicates)

1013 normalized reads at pioneered TFAP2-activated promoters. (F-F') Violin plots

1014 representing (F) anti-H3K27Ac (F') ATAC-seq and (E') anti-H3K4Me3 normalized

1015 reads at non-pioneered TFAP2-activated promoters. (G-G') Violin plots representing

1016 (G) anti-H3K27Ac (G') ATAC-seq and (G') anti-H3K4Me3 normalized reads at

1017 pioneered TFAP2-inhibited promoters. (H-H') Violin plots representing (H) anti-

1018 H3K27Ac (H') ATAC-seq and (H') anti-H3K4Me3 normalized reads at non-

1019 pioneered TFAP2-inhibited promoters. P-values shown were determined by Students

1020 t-test. (I-L) Hypergeometric analysis of TFAP2 regulated enhancers at TFAP2-

1021 activated $\left({ }^{*}\right)$ and TFAP2-inhibited $\left({ }^{* *}\right)$ genes in TFAP2-KO cells (FDR $<0.05$,

$1022|\log 2 \mathrm{FC}|>1)$. The number of promoters in each category of TFAP2-regulated

1023 promoters is shown.

1024

1025 Figure S7: Additional examples of TFAP2-activated and -inhibited promoters.

1026 (A-B) Screenshots of IGV genome browser (GRCH37/hg19) visualizing anti-

1027 TFAP2A, anti-H3K4Me3, anti-H3K27Ac CUT\&RUN-seq, ATAC-seq and RNA-seq

1028 profiles at (A) the TFAP2-activated ZNF540 promoter and (B) the TFAP2-inhibited

1029 S100A16 promoter. Genotypes as labeled; y-axes are grouped scaled per dataset.

1031 Figure S8: TFAP2 paralogs modulate the binding of MITF. (A) Density heatmap

1032 centred on 9,413 peaks co-bound by TFAP2A and MITF, showing anti-TFAP2, anti-

1033 MITF, ATAC-seq, and anti-H3K27Ac CUT\&RUN profiles in SK-MEL-28 cells. 
1034 Regions shown are +/- $5 \mathrm{~kb}$ from peak center. (B) Violin plot showing anti-MITF 1035 CUT\&RUN signal (RPKM) in TFAP2-KO and SK-MEL-28 cells at loci not bound by

1036 TFAP2A. ns; non-significant by Students t-test. (C) Scatterplot of TFAP2-dependent

1037 MITF peaks showing log2 normalized reads on the $x$-axis and log2FC on the y-axis

1038 in TFAP2-KO versus WT cells. (D) Screenshots of IGV genome browser

1039 (GRCH37/hg19); genotypes as labeled, visualizing anti-TFAP2A and anti-MITF

1040 CUT\&RUN-seq and ATAC-seq profiles at TFAP2-dependent MITF peaks, mutually

1041 dependent MITF/TFAP2 peaks and an example of a non-overlapping, TFAP2-

1042 independent MITF peak. (E) Volcano plot showing increased H3K27Me3 CUT\&RUN

1043 signal at mutually dependent TFAP2/MITF peaks in MITF-KO cells versus WT cells.

1044 P-values were determined by Students t-test. Normalized reads (RPKM), (F-G)

1045 Screenshots of IGV genome browser (GRCH37/hg19), visualizing anti-H3K27Me3

1046 CUT\&RUN-seq profiles in MITF-KO and WT cells. Peaks were called using MACS2

1047 software (two independent replicates) and are illustrated by blue bars. Two examples

1048 loci of mutually dependent TFAP2/MITF peaks showing increased H3K27Me3

1049 signals are shown, (F) at the TRPM1 promoter and (G) at two FRMD4B enhancers.

1050 Mutually-dependent peaks are indicated by red arrows.

1052 Figure S9: Density heatmap of TFAP2 regulated MITF peaks. Density heatmap 1053 centred on TFAP2 regulated MITF peaks (Top cluster) TFAP2-dependent MITF 1054 peaks, (Second cluster) Mutually dependent TFAP2/MITF peaks, (third cluster)

1055 TFAP2-inhibited MITF peaks and (forth cluster) independent peaks, showing two 1056 replicates of anti-MITF CUT\&RUN in SK-MEL-28 and TFAP2-KO cells, and two 1057 replicates of anti-TFAP2A CUT\&RUN in SK-MEL-28 and MITF-KO cells. Regions 1058 shown are $+/-5 \mathrm{~kb}$ from peak center.

1060 Figure S10: TFAP2 and MITF do not co-inhibit enhancers at TFAP2-inhibited or 1061 MITF-inhibited genes in SK-MEL-28 cells. (A-A') Violin plot of TFAP2 dependent MITF peaks at TFAP2-inhibied enhancers (i.e. co-inhibited enhancers) showing (B) anti-MITF CUT\&RUN and (A') ATAC-Seq profiles in TFAP2-KO and WT SK-MEL-28

1064 cells. Such loci were not significantly enriched at MITF-inhibited or TFAP2-inhibited 1065 genes. (A") Schematic of TFAP2/ MITF co-inhibited enhancers. In this example 1066 TFAP2 is a pioneer factor recruiting MITF, in its repressor form, to condense 1067 chromatin. Loss of TFAP2 in TFAP2-KO cells results in loss of MITF-repressor 
binding and opening of chromatin by an alternative pioneer factor. (B-B') Violin plot of TFAP2-inhibited MITF peaks at modestly TFAP2-inhibted enhancers showing (C) anti-MITF CUT\&RUN (B') ATAC-Seq profiles in TFAP2-KO and WT SK-MEL-28 cells. Such loci were not significantly enriched at MITF-inhibited or TFAP2-inhibited genes. (B") Schematic of TFAP2-inhibted MITF peaks at modestly inhibited enhancers (Log2FC > $-0.5--1$ ). In this example TFAP2 recruits a repressor protein and inhibits MITF binding in WT cells. In the absence of TFAP2, MITF recruits SWI/SNF and opens chromatin via an alternative pioneer factor.

Figure S11: Genes that harbor TFAP2-independent anti-MITF peaks are enriched for cell cycle and DNA-repair. (A) Plot-profile showing MITF CUT\&RUN peak signal at TFAP2-independent MITF peaks in TFAP2-KO and WT SK-MEL-28 cell lines. (B) Genes that harbor TFAP2-independent anti-MITF peaks were analyzed for enriched gene ontology biological process using GREAT (single nearest gene +/100kb).

Figure S12: Example of an TFAP2-dependent enhancer at intron 2 of MITF. Screenshot of IGV genome browser (GRCH37/hg19) visualizing anti-TFAP2A, ATAC-seq, anti-H3K27Ac and RNA-seq profiles at intron 2 of MITF. Dashed rectangle indicates an TFAP2-dependent NDR. MITF and downstream regions are shown, blue arrows indicate strand orientation and horizontal rectangles the exons. Genotypes are as labeled; y-axes are grouped scaled per dataset.

Figure S13: TFAP2 directly activates genes associated with cell differentiation and proliferation, and direct inhibits genes associated with cell adhesion and cell migration. (A-B) The top 55 genes that are directly TFAP2-activated and associated with the GO terms (A) cell differentiation and pigmentation and (B) proliferation are represented by heatmap (log2FC). (C) the top 55 genes that are directly TFAP2-inhibited and associated with the GO terms cell adhesion and cell migration are represented by heatmap (log2FC). C1: TFAP2-KO clone 4.3, C2: TFAP2-KO clone 2.12. (D) Wound healing scratch-recovery-assay over 24 hours in WT and TFAP2-KO cells. TFAP2-KO cells show reduced migration capacity compared to wild type SK-MEL-28 cells. 


\section{References cited:}

Aras, S., Saladi, S.V., Basuroy, T., Marathe, H.G., Lores, P., de la Serna, I.L., 2019. BAF60A mediates interactions between the microphthalmia-associated transcription factor and the BRG1-containing SWI/SNF complex during melanocyte differentiation. J Cell Physiol 234, 11780-11791. Atchison, M.L., 2014. Function of YY1 in Long-Distance DNA Interactions. Front Immunol $1110 \quad 5,45$.

1111 Bamforth, S.D., Braganca, J., Eloranta, J.J., Murdoch, J.N., Marques, F.I., Kranc, K.R., 1112 Farza, H., Henderson, D.J., Hurst, H.C., Bhattacharya, S., 2001. Cardiac malformations, 1113 adrenal agenesis, neural crest defects and exencephaly in mice lacking Cited2, a new Tfap2 co-activator. Nat Genet 29, 469-474. Barrallo-Gimeno, A., Holzschuh, J., Driever, W., Knapik, E.W., 2004. Neural crest survival and differentiation in zebrafish depends on mont blanc/tfap2a gene function. Development 131, 1463-1477. Baxter, L.L., Loftus, S.K., Pavan, W.J., 2009. Networks and pathways in pigmentation, health, and disease. Wiley Interdiscip Rev Syst Biol Med 1, 359-371. Baxter, L.L., Watkins-Chow, D.E., Pavan, W.J., Loftus, S.K., 2019. A curated gene list for expanding the horizons of pigmentation biology. Pigment Cell Melanoma Res 32, 348-358. Bejjani, F., Evanno, E., Zibara, K., Piechaczyk, M., Jariel-Encontre, I., 2019. The AP-1 transcriptional complex: Local switch or remote command? Biochim Biophys Acta Rev Cancer 1872, 11-23.

Betancur, P., Bronner-Fraser, M., Sauka-Spengler, T., 2010. Assembling neural crest regulatory circuits into a gene regulatory network. Annu Rev Cell Dev Biol 26, 581-603. Braganca, J., Eloranta, J.J., Bamforth, S.D., Ibbitt, J.C., Hurst, H.C., Bhattacharya, S., 2003. Physical and functional interactions among AP-2 transcription factors, p300/CREB-binding protein, and CITED2. The Journal of biological chemistry 278, 16021-16029. Brewer, S., Jiang, X., Donaldson, S., Williams, T., Sucov, H.M., 2002. Requirement for AP2alpha in cardiac outflow tract morphogenesis. Mech Dev 110, 139-149. Buenrostro, J.D., Giresi, P.G., Zaba, L.C., Chang, H.Y., Greenleaf, W.J., 2013. Transposition of native chromatin for fast and sensitive epigenomic profiling of open chromatin, DNAbinding proteins and nucleosome position. Nat Methods 10, 1213-1218. Buenrostro, J.D., Wu, B., Chang, H.Y., Greenleaf, W.J., 2015. ATAC-seq: A Method for Assaying Chromatin Accessibility Genome-Wide. Curr Protoc Mol Biol 109, 21.29.2121.29.29. Ferretti, L., Huang, T.-H., Garg, M., Nsengimana, J., Montal, E., Tagore, M., Hunter, M., Newton-Bishop, J., Middleton, M.R., Corrie, P., Adams, D.J., Rabbie, R., Levesque, M.P., Cornell, R.A., Yanai, I., Xavier, J.B., White, R.M., 2021. Cell state diversity promotes metastasis through heterotypic cluster formation in melanoma. Developmental Cell, 2020.2008.2024.265140.

Carreira, S., Goodall, J., Denat, L., Rodriguez, M., Nuciforo, P., Hoek, K.S., Testori, A., Larue, L., Goding, C.R., 2006. Mitf regulation of Dial controls melanoma proliferation and invasiveness. Genes Dev 20, 3426-3439.

Creyghton, M.P., Cheng, A.W., Welstead, G.G., Kooistra, T., Carey, B.W., Steine, E.J., Hanna, J., Lodato, M.A., Frampton, G.M., Sharp, P.A., Boyer, L.A., Young, R.A., Jaenisch, 
1149 R., 2010. Histone H3K27ac separates active from poised enhancers and predicts

1150 developmental state. Proc Natl Acad Sci U S A 107, 21931-21936.

1151 de la Serna, I.L., Ohkawa, Y., Higashi, C., Dutta, C., Osias, J., Kommajosyula, N.,

1152 Tachibana, T., Imbalzano, A.N., 2006. The microphthalmia-associated transcription factor

1153 requires SWI/SNF enzymes to activate melanocyte-specific genes. J Biol Chem 281, 20233-

\section{1.}

1155 Dilshat, R., Fock, V., Kenny, C., Gerritsen, I., Lasseur, R.M.J., Travnickova, J., Eichhoff, O.M., Cerny, P., Moller, K., Sigurbjornsdottir, S., Kirty, K., Einarsdottir, B.O., Cheng, P.F., Levesque, M., Cornell, R.A., Patton, E.E., Larue, L., de Tayrac, M., Magnusdottir, E., Helga Ogmundsdottir, M., Steingrimsson, E., 2021. MITF reprograms the extracellular matrix and

1159 focal adhesion in melanoma. Elife 10.

1160 Dobin, A., Davis, C.A., Schlesinger, F., Drenkow, J., Zaleski, C., Jha, S., Batut, P., Chaisson, M., Gingeras, T.R., 2013. STAR: ultrafast universal RNA-seq aligner. Bioinformatics 29, 1521. $\mathrm{Lu}, \mathrm{J} ., 2012$. FOXC1, a target of polycomb, inhibits metastasis of breast cancer cells. Breast Cancer Res Treat 131, 65-73.

1168 Fernandez Garcia, M., Moore, C.D., Schulz, K.N., Alberto, O., Donague, G., Harrison, M.M., 1169 Zhu, H., Zaret, K.S., 2019. Structural Features of Transcription Factors Associating with

1170 Nucleosome Binding. Mol Cell 75, 921-932 e926.

1171 Goding, C.R., 2000. Mitf from neural crest to melanoma: signal transduction and transcription in the melanocyte lineage. Genes Dev 14, 1712-1728.

1173 Grossman, S.R., Engreitz, J., Ray, J.P., Nguyen, T.H., Hacohen, N., Lander, E.S., 2018.

1174 Positional specificity of different transcription factor classes within enhancers. Proc Natl

1175 Acad Sci U S A 115, E7222-E7230. Hartman, M.L., Czyz, M., 2015. MITF in melanoma: mechanisms behind its expression and activity. Cell Mol Life Sci 72, 1249-1260.

1178 Higdon, C.W., Mitra, R.D., Johnson, S.L., 2013. Gene expression analysis of zebrafish melanocytes, iridophores, and retinal pigmented epithelium reveals indicators of biological function and developmental origin. PLoS One 8, e67801.

1181 Hoek, K.S., Eichhoff, O.M., Schlegel, N.C., Döbbeling, U., Kobert, N., Schaerer, L., Hemmi,

1182 S., Dummer, R., 2008a. In vivo switching of human melanoma cells between proliferative and invasive states. Cancer Res 68, 650-656.

Hoek, K.S., Schlegel, N.C., Brafford, P., Sucker, A., Ugurel, S., Kumar, R., Weber, B.L., Nathanson, K.L., Phillips, D.J., Herlyn, M., Schadendorf, D., Dummer, R., 2006. Metastatic potential of melanomas defined by specific gene expression profiles with no BRAF signature.

1188 Hoek, K.S., Schlegel, N.C., Eichhoff, O.M., Widmer, D.S., Praetorius, C., Einarsson, S.O., 1189 Valgeirsdottir, S., Bergsteinsdottir, K., Schepsky, A., Dummer, R., Steingrimsson, E., $2008 \mathrm{~b}$. 1190 Novel MITF targets identified using a two-step DNA microarray strategy. Pigment Cell 1191 Melanoma Res 21, 665-676.

1192 Huang, S., Jean, D., Luca, M., Tainsky, M.A., Bar-Eli, M., 1998. Loss of AP-2 results in downregulation of c-KIT and enhancement of melanoma tumorigenicity and metastasis. EMBO J 17, 4358-4369.

Jonsson, G., Busch, C., Knappskog, S., Geisler, J., Miletic, H., Ringner, M., Lillehaug, J.R., Borg, A., Lonning, P.E., 2010. Gene expression profiling-based identification of molecular subtypes in stage IV melanomas with different clinical outcome. Clin Cancer Res 16, 33563367. 
1199 Keenen, B., Qi, H., Saladi, S.V., Yeung, M., de la Serna, I.L., 2010. Heterogeneous

1200 SWI/SNF chromatin remodeling complexes promote expression of microphthalmiaassociated transcription factor target genes in melanoma. Oncogene 29, 81-92. Kim, S., Yu, N.K., Kaang, B.K., 2015. CTCF as a multifunctional protein in genome regulation and gene expression. Exp Mol Med 47, e166. Knight, R.D., Javidan, Y., Nelson, S., Zhang, T., Schilling, T., 2004. Skeletal and pigment cell defects in the lockjaw mutant reveal multiple roles for zebrafish tfap2a in neural crest development. Dev Dyn 229, 87-98. Knight, R.D., Nair, S., Nelson, S.S., Afshar, A., Javidan, Y., Geisler, R., Rauch, G.J., Schilling, T.F., 2003. lockjaw encodes a zebrafish tfap2a required for early neural crest development. Development 130, 5755-5768. Kołat, D., Kałuzińska, Ż., Bednarek, A.K., Płuciennik, E., 2021. WWOX Loses the Ability to Regulate Oncogenic AP-2 $\gamma$ and Synergizes with Tumor Suppressor AP-2 $\alpha$ in High-Grade Bladder Cancer. Cancers (Basel) 13.

Kuckenberg, P., Kubaczka, C., Schorle, H., 2012. The role of transcription factor Tcfap2c/TFAP2C in trophectoderm development. Reprod Biomed Online 25, 12-20. Lang, D., Lu, M.M., Huang, L., Engleka, K.A., Zhang, M., Chu, E.Y., Lipner, S., Skoultchi, A., Millar, S.E., Epstein, J.A., 2005. Pax3 functions at a nodal point in melanocyte stem cell differentiation. Nature 433, 884-887.

1218 Langmead, B., Salzberg, S.L., 2012. Fast gapped-read alignment with Bowtie 2. Nat Methods 1219 9, 357-359.

1220 Langmead, B., Trapnell, C., Pop, M., Salzberg, S.L., 2009. Ultrafast and memory-efficient alignment of short DNA sequences to the human genome. Genome Biol 10, R25. Laurette, P., Strub, T., Koludrovic, D., Keime, C., Le Gras, S., Seberg, H., Van Otterloo, E., Imrichova, H., Siddaway, R., Aerts, S., Cornell, R.A., Mengus, G., Davidson, I., 2015. Transcription factor MITF and remodeller BRG1 define chromatin organisation at regulatory elements in melanoma cells. Elife 4.

Lee, B.K., Uprety, N., Jang, Y.J., Tucker, S.K., Rhee, C., LeBlanc, L., Beck, S., Kim, J., 2018. Fosl1 overexpression directly activates trophoblast-specific gene expression programs in embryonic stem cells. Stem Cell Res 26, 95-102. TP53 mutation status in human cancer cell lines: a reassessment. Hum Mutat 35, 756-765. Li, L., Wang, Y., Torkelson, J.L., Shankar, G., Pattison, J.M., Zhen, H.H., Fang, F., Duren, Z., Xin, J., Gaddam, S., Melo, S.P., Piekos, S.N., Li, J., Liaw, E.J., Chen, L., Li, R., Wernig, M., Wong, W.H., Chang, H.Y., Oro, A.E., 2019. TFAP2C- and p63-Dependent Networks Sequentially Rearrange Chromatin Landscapes to Drive Human Epidermal Lineage Commitment. Cell Stem Cell 24, 271-284 e278.

Li, W., Cornell, R.A., 2007. Redundant activities of Tfap2a and Tfap2c are required for neural crest induction and development of other non-neural ectoderm derivatives in zebrafish embryos. Dev Biol 304, 338-354.

1239 Lin, C.Y., Chao, A., Wang, T.H., Lee, L.Y., Yang, L.Y., Tsai, C.L., Wang, H.S., Lai, C.H., 1240 2016. Nucleophosmin/B23 is a negative regulator of estrogen receptor alpha expression via AP2gamma in endometrial cancer cells. Oncotarget 7, 60038-60052. Liu, H., Duncan, K., Helverson, A., Kumari, P., Mumm, C., Xiao, Y., Carlson, J.C., Darbellay, F., Visel, A., Leslie, E., Breheny, P., Erives, A.J., Cornell, R.A., 2020. Analysis of zebrafish periderm enhancers facilitates identification of a regulatory variant near human KRT8/18. Elife 9.

Liu, H., Tan, B.C., Tseng, K.H., Chuang, C.P., Yeh, C.W., Chen, K.D., Lee, S.C., Yung, B.Y., 2007. Nucleophosmin acts as a novel AP2alpha-binding transcriptional corepressor during cell differentiation. EMBO Rep 8, 394-400. 
Love, M.I., Huber, W., Anders, S., 2014. Moderated estimation of fold change and dispersion for RNA-seq data with DESeq2. Genome Biol 15, 550. Luo, T., Matsuo-Takasaki, M., Thomas, M.L., Weeks, D.L., Sargent, T.D., 2002. Transcription factor AP-2 is an essential and direct regulator of epidermal development in Xenopus. Dev Biol 245, 136-144. Mavrothalassitis, G., Ghysdael, J., 2000. Proteins of the ETS family with transcriptional repressor activity. Oncogene 19, 6524-6532. Mayran, A., Khetchoumian, K., Hariri, F., Pastinen, T., Gauthier, Y., Balsalobre, A., Drouin, J., 2018. Pioneer factor Pax7 deploys a stable enhancer repertoire for specification of cell fate. Nat Genet 50, 259-269. McLean, C.Y., Bristor, D., Hiller, M., Clarke, S.L., Schaar, B.T., Lowe, C.B., Wenger, A.M., Bejerano, G., 2010. GREAT improves functional interpretation of cis-regulatory regions. Nat Biotechnol 28, 495-501.

Meers, M.P., Bryson, T.D., Henikoff, J.G., Henikoff, S., 2019. Improved CUT\&RUN chromatin profiling tools. Elife 8.

Mi, H., Ebert, D., Muruganujan, A., Mills, C., Albou, L.P., Mushayamaha, T., Thomas, P.D., 2021. PANTHER version 16: a revised family classification, tree-based classification tool, enhancer regions and extensive API. Nucleic Acids Res 49, D394-d403. Miller, J.C., Holmes, M.C., Wang, J., Guschin, D.Y., Lee, Y.L., Rupniewski, I., Beausejour, C.M., Waite, A.J., Wang, N.S., Kim, K.A., Gregory, P.D., Pabo, C.O., Rebar, E.J., 2007. An improved zinc-finger nuclease architecture for highly specific genome editing. Nat Biotechnol 25, 778-785. Mitchell, P.J., Timmons, P.M., Hebert, J.M., Rigby, P.W., Tjian, R., 1991. Transcription factor AP-2 is expressed in neural crest cell lineages during mouse embryogenesis. Genes Dev 5, 105-119.

Mollaaghababa, R., Pavan, W.J., 2003. The importance of having your SOX on: role of SOX10 in the development of neural crest-derived melanocytes and glia. Oncogene 22, 30243034. beta gene expression during murine embryogenesis. Developmental Dynamics 208, 115-124. Pastor, W.A., Liu, W., Chen, D., Ho, J., Kim, R., Hunt, T.J., Lukianchikov, A., Liu, X., Polo, J.M., Jacobsen, S.E., Clark, A.T., 2018. TFAP2C regulates transcription in human naive pluripotency by opening enhancers. Nat Cell Biol 20, 553-564. Pekowska, A., Benoukraf, T., Zacarias-Cabeza, J., Belhocine, M., Koch, F., Holota, H., Imbert, J., Andrau, J.C., Ferrier, P., Spicuglia, S., 2011. H3K4 tri-methylation provides an epigenetic signature of active enhancers. EMBO J 30, 4198-4210.

Petruk, S., Cai, J., Sussman, R., Sun, G., Kovermann, S.K., Mariani, S.A., Calabretta, B., McMahon, S.B., Brock, H.W., Iacovitti, L., Mazo, A., 2017. Delayed Accumulation of H3K27me3 on Nascent DNA Is Essential for Recruitment of Transcription Factors at Early Stages of Stem Cell Differentiation. Mol Cell 66, 247-257 e245. Pihlajamaa, P., Sahu, B., Lyly, L., Aittomaki, V., Hautaniemi, S., Janne, O.A., 2014. Tissuespecific pioneer factors associate with androgen receptor cistromes and transcription programs. EMBO J 33, 312-326.

Praetorius, C., Grill, C., Stacey, S.N., Metcalf, A.M., Gorkin, D.U., Robinson, K.C., Van Otterloo, E., Kim, R.S., Bergsteinsdottir, K., Ogmundsdottir, M.H., Magnusdottir, E., Mishra, P.J., Davis, S.R., Guo, T., Zaidi, M.R., Helgason, A.S., Sigurdsson, M.I., Meltzer, P.S., Merlino, G., Petit, V., Larue, L., Loftus, S.K., Adams, D.R., Sobhiafshar, U., Emre, N.C., Pavan, W.J., Cornell, R., Smith, A.G., McCallion, A.S., Fisher, D.E., Stefansson, K., Sturm, R.A., Steingrimsson, E., 2013. A polymorphism in IRF4 affects human pigmentation through a tyrosinase-dependent MITF/TFAP2A pathway. Cell 155, 1022-1033. 
1299 Rambow, F., Job, B., Petit, V., Gesbert, F., Delmas, V., Seberg, H., Meurice, G., Van

1300 Otterloo, E., Dessen, P., Robert, C., Gautheret, D., Cornell, R.A., Sarasin, A., Larue, L.,

1301 2015. New Functional Signatures for Understanding Melanoma Biology from Tumor Cell

1302 Lineage-Specific Analysis. Cell Rep 13, 840-853.

1303 Rambow, F., Marine, J.C., Goding, C.R., 2019. Melanoma plasticity and phenotypic diversity: therapeutic barriers and opportunities. Genes Dev 33, 1295-1318. Rambow, F., Rogiers, A., Marin-Bejar, O., Aibar, S., Femel, J., Dewaele, M., Karras, P., Brown, D., Chang, Y.H., Debiec-Rychter, M., Adriaens, C., Radaelli, E., Wolter, P., Bechter, O., Dummer, R., Levesque, M., Piris, A., Frederick, D.T., Boland, G., Flaherty, K.T., van den Oord, J., Voet, T., Aerts, S., Lund, A.W., Marine, J.C., 2018. Toward Minimal Residual Disease-Directed Therapy in Melanoma. Cell 174, 843-855 e819. Ramírez, F., Ryan, D.P., Grüning, B., Bhardwaj, V., Kilpert, F., Richter, A.S., Heyne, S., Dündar, F., Manke, T., 2016. deepTools2: a next generation web server for deep-sequencing data analysis. Nucleic Acids Res 44, W160-165.

Ringrose, L., Paro, R., 2004. Epigenetic regulation of cellular memory by the Polycomb and Trithorax group proteins. Annu Rev Genet 38, 413-443.

Ross-Innes, C.S., Stark, R., Teschendorff, A.E., Holmes, K.A., Ali, H.R., Dunning, M.J., Brown, G.D., Gojis, O., Ellis, I.O., Green, A.R., Ali, S., Chin, S.F., Palmieri, C., Caldas, C., Carroll, J.S., 2012. Differential oestrogen receptor binding is associated with clinical outcome in breast cancer. Nature 481, 389-393. Sander, J.D., Dahlborg, E.J., Goodwin, M.J., Cade, L., Zhang, F., Cifuentes, D., Curtin, S.J., Blackburn, J.S., Thibodeau-Beganny, S., Qi, Y., Pierick, C.J., Hoffman, E., Maeder, M.L., Khayter, C., Reyon, D., Dobbs, D., Langenau, D.M., Stupar, R.M., Giraldez, A.J., Voytas, D.F., Peterson, R.T., Yeh, J.R., Joung, J.K., 2011. Selection-free zinc-finger-nuclease engineering by context-dependent assembly (CoDA). Nat Methods 8, 67-69.

Sander, J.D., Maeder, M.L., Reyon, D., Voytas, D.F., Joung, J.K., Dobbs, D., 2010. ZiFiT (Zinc Finger Targeter): an updated zinc finger engineering tool. Nucleic Acids Res 38, W462-468.

Schorle, H., Meier, P., Buchert, M., Jaenisch, R., Mitchell, P.J., 1996. Transcription factor AP-2 essential for cranial closure and craniofacial development. Nature 381, 235-238. Seberg, H.E., Van Otterloo, E., Cornell, R.A., 2017a. Beyond MITF: Multiple transcription factors directly regulate the cellular phenotype in melanocytes and melanoma. Pigment Cell Melanoma Res 30, 454-466.

Seberg, H.E., Van Otterloo, E., Loftus, S.K., Liu, H., Bonde, G., Sompallae, R., Gildea, D.E., Santana, J.F., Manak, J.R., Pavan, W.J., Williams, T., Cornell, R.A., 2017b. TFAP2 paralogs regulate melanocyte differentiation in parallel with MITF. PLoS Genet 13, e1006636.

Sekiya, T., Zaret, K.S., 2007. Repression by Groucho/TLE/Grg proteins: genomic site recruitment generates compacted chromatin in vitro and impairs activator binding in vivo. Mol Cell 28, 291-303.

Sherwood, R.I., Hashimoto, T., O'Donnell, C.W., Lewis, S., Barkal, A.A., van Hoff, J.P., Karun, V., Jaakkola, T., Gifford, D.K., 2014. Discovery of directional and nondirectional pioneer transcription factors by modeling DNase profile magnitude and shape. Nat Biotechnol 32, 171-178.

Skene, P.J., Henikoff, S., 2017. An efficient targeted nuclease strategy for high-resolution mapping of DNA binding sites. Elife 6.

Strub, T., Giuliano, S., Ye, T., Bonet, C., Keime, C., Kobi, D., Le Gras, S., Cormont, M., Ballotti, R., Bertolotto, C., Davidson, I., 2011. Essential role of microphthalmia transcription factor for DNA replication, mitosis and genomic stability in melanoma. Oncogene 30, 23192332. 
Swinstead, E.E., Miranda, T.B., Paakinaho, V., Baek, S., Goldstein, I., Hawkins, M., Karpova, T.S., Ball, D., Mazza, D., Lavis, L.D., Grimm, J.B., Morisaki, T., Grontved, L., Presman, D.M., Hager, G.L., 2016a. Steroid Receptors Reprogram FoxA1 Occupancy through Dynamic Chromatin Transitions. Cell 165, 593-605. Swinstead, E.E., Paakinaho, V., Presman, D.M., Hager, G.L., 2016b. Pioneer factors and ATP-dependent chromatin remodeling factors interact dynamically: A new perspective: Multiple transcription factors can effect chromatin pioneer functions through dynamic interactions with ATP-dependent chromatin remodeling factors. Bioessays 38, 1150-1157. Tan, C.C., Sindhu, K.V., Li, S., Nishio, H., Stoller, J.Z., Oishi, K., Puttreddy, S., Lee, T.J., Epstein, J.A., Walsh, M.J., Gelb, B.D., 2008. Transcription factor Ap2delta associates with Ash21 and ALR, a trithorax family histone methyltransferase, to activate Hoxc8 transcription. Proc Natl Acad Sci U S A 105, 7472-7477. Tan, S.K., Lin, Z.H., Chang, C.W., Varang, V., Chng, K.R., Pan, Y.F., Yong, E.L., Sung, W.K., Cheung, E., 2011. AP-2gamma regulates oestrogen receptor-mediated long-range chromatin interaction and gene transcription. EMBO J 30, 2569-2581. Tirosh, I., Izar, B., Prakadan, S.M., Wadsworth, M.H., 2nd, Treacy, D., Trombetta, J.J., Rotem, A., Rodman, C., Lian, C., Murphy, G., Fallahi-Sichani, M., Dutton-Regester, K., Lin, J.R., Cohen, O., Shah, P., Lu, D., Genshaft, A.S., Hughes, T.K., Ziegler, C.G., Kazer, S.W., Gaillard, A., Kolb, K.E., Villani, A.C., Johannessen, C.M., Andreev, A.Y., Van Allen, E.M., Bertagnolli, M., Sorger, P.K., Sullivan, R.J., Flaherty, K.T., Frederick, D.T., Jane-Valbuena, J., Yoon, C.H., Rozenblatt-Rosen, O., Shalek, A.K., Regev, A., Garraway, L.A., 2016. Dissecting the multicellular ecosystem of metastatic melanoma by single-cell RNA-seq. Science 352, 189-196. Tsoi, J., Robert, L., Paraiso, K., Galvan, C., Sheu, K.M., Lay, J., Wong, D.J.L., Atefi, M., Shirazi, R., Wang, X., Braas, D., Grasso, C.S., Palaskas, N., Ribas, A., Graeber, T.G., 2018. Multi-stage Differentiation Defines Melanoma Subtypes with Differential Vulnerability to Drug-Induced Iron-Dependent Oxidative Stress. Cancer Cell 33, 890-904 e895. Van Otterloo, E., Li, W., Bonde, G., Day, K.M., Hsu, M.Y., Cornell, R.A., 2010. Differentiation of zebrafish melanophores depends on transcription factors AP2 alpha and AP2 epsilon. PLoS Genet 6. Van Otterloo, E., Li, W., Garnett, A., Cattell, M., Medeiros, D.M., Cornell, R.A., 2012. Novel Tfap2-mediated control of soxE expression facilitated the evolutionary emergence of the neural crest. Development 139, 720-730. Verfaillie, A., Imrichova, H., Atak, Z.K., Dewaele, M., Rambow, F., Hulselmans, G., Christiaens, V., Svetlichnyy, D., Luciani, F., Van den Mooter, L., Claerhout, S., Fiers, M., Journe, F., Ghanem, G.E., Herrmann, C., Halder, G., Marine, J.C., Aerts, S., 2015. Decoding the regulatory landscape of melanoma reveals TEADS as regulators of the invasive cell state. Nat Commun 6, 6683.

Vierbuchen, T., Ling, E., Cowley, C.J., Couch, C.H., Wang, X., Harmin, D.A., Roberts, C.W.M., Greenberg, M.E., 2017. AP-1 Transcription Factors and the BAF Complex Mediate Signal-Dependent Enhancer Selection. Mol Cell 68, 1067-1082.e1012. Voss, T.C., Hager, G.L., 2014. Dynamic regulation of transcriptional states by chromatin and transcription factors. Nat Rev Genet 15, 69-81. Wang, X., Bolotin, D., Chu, D.H., Polak, L., Williams, T., Fuchs, E., 2006. AP-2alpha: a regulator of EGF receptor signaling and proliferation in skin epidermis. J Cell Biol 172, 409421.

Wang, X., Pasolli, H.A., Williams, T., Fuchs, E., 2008. AP-2 factors act in concert with Notch to orchestrate terminal differentiation in skin epidermis. J Cell Biol 183, 37-48. Watts, J.A., Zhang, C., Klein-Szanto, A.J., Kormish, J.D., Fu, J., Zhang, M.Q., Zaret, K.S., 2011. Study of FoxA pioneer factor at silent genes reveals RfX-repressed enhancer at Cdx2 
1398 and a potential indicator of esophageal adenocarcinoma development. PLoS Genet 7,

1399 e1002277.

1400 White, J.R., Thompson, D.T., Koch, K.E., Kiriazov, B.S., Beck, A.C., van der Heide, D.M., 1401 Grimm, B.G., Kulak, M.V., Weigel, R.J., 2021. AP-2alpha-mediated activation of E2F and 1402 EZH2 drives melanoma metastasis. Cancer Res.

1403 Wilson, B.G., Wang, X., Shen, X., McKenna, E.S., Lemieux, M.E., Cho, Y.J., Koellhoffer, 1404 E.C., Pomeroy, S.L., Orkin, S.H., Roberts, C.W., 2010. Epigenetic antagonism between 1405 polycomb and SWI/SNF complexes during oncogenic transformation. Cancer Cell 18, 3161406328.

1407 Wong, P.P., Miranda, F., Chan, K.V., Berlato, C., Hurst, H.C., Scibetta, A.G., 2012. Histone 1408 demethylase KDM5B collaborates with TFAP2C and Myc to repress the cell cycle inhibitor 1409 p21(cip) (CDKN1A). Mol Cell Biol 32, 1633-1644.

1410 Yu, G., Wang, L.G., Han, Y., He, Q.Y., 2012. clusterProfiler: an R package for comparing 1411 biological themes among gene clusters. Omics 16, 284-287.

1412 Zaret, K.S., 2020. Pioneer Transcription Factors Initiating Gene Network Changes. Annu Rev 1413 Genet 54, 367-385.

1414 Zaret, K.S., Carroll, J.S., 2011. Pioneer transcription factors: establishing competence for 1415 gene expression. Genes Dev 25, 2227-2241.

1416 Zhang, Y., Liu, T., Meyer, C.A., Eeckhoute, J., Johnson, D.S., Bernstein, B.E., Nusbaum, C., 1417 Myers, R.M., Brown, M., Li, W., Liu, X.S., 2008. Model-based analysis of ChIP-Seq

1418 (MACS). Genome Biol 9, R137. 